\title{
Intraspecific comparative genomics of isolates of the Norway spruce pathogen (Heterobasidion parviporum) and identification of its potential virulence factors
}

\author{
Zhen Zeng ${ }^{1}$, Hui Sun ${ }^{1,2}$, Eeva J. Vainio ${ }^{3}$, Tommaso Raffaello ${ }^{1}$, Andriy Kovalchuk', Emmanuelle Morin ${ }^{4}$,
} Sébastien Duplessis ${ }^{4,5}$ and Fred O. Asiegbu ${ }^{1^{*}}$

\begin{abstract}
Background: Heterobasidion parviporum is an economically most important fungal forest pathogen in northern Europe, causing root and butt rot disease of Norway spruce (Picea abies (L.) Karst.). The mechanisms underlying the pathogenesis and virulence of this species remain elusive. No reference genome to facilitate functional analysis is available for this species.

Results: To better understand the virulence factor at both phenotypic and genomic level, we characterized $15 \mathrm{H}$. parviporum isolates originating from different locations across Finland for virulence, vegetative growth, sporulation and saprotrophic wood decay. Wood decay capability and latitude of fungal origins exerted interactive effects on their virulence and appeared important for $\mathrm{H}$. parviporum virulence. We sequenced the most virulent isolate, the first full genome sequences of $\mathrm{H}$. parviporum as a reference genome, and re-sequenced the remaining $14 \mathrm{H}$. parviporum isolates. Genome-wide alignments and intrinsic polymorphism analysis showed that these isolates exhibited overall high genomic similarity with an average of at least $96 \%$ nucleotide identity when compared to the reference, yet had remarkable intra-specific level of polymorphism with a bias for CpG to TpG mutations. Reads mapping coverage analysis enabled the classification of all predicted genes into five groups and uncovered two genomic regions exclusively present in the reference with putative contribution to its higher virulence. Genes enriched for copy number variations (deletions and duplications) and nucleotide polymorphism were involved in oxidationreduction processes and encoding domains relevant to transcription factors. Some secreted protein coding genes based on the genome-wide selection pressure, or the presence of variants were proposed as potential virulence candidates.
\end{abstract}

Conclusion: Our study reported on the first reference genome sequence for this Norway spruce pathogen $(H$. parviporum). Comparative genomics analysis gave insight into the overall genomic variation among this fungal species and also facilitated the identification of several secreted protein coding genes as putative virulence factors for the further functional analysis. We also analyzed and identified phenotypic traits potentially linked to its virulence.

Keywords: Heterobasidion parviporum, Comparative genomics, Virulence factors, Secreted proteins, CpG-biased mutation, Saprotrophic wood decay, Oxidation-reduction process, Transcription factors

\footnotetext{
* Correspondence: fred.asiegbu@helsinki.fi

${ }^{1}$ Department of Forest Sciences, University of Helsinki, Helsinki, Finland

Full list of author information is available at the end of the article
} 


\section{Background}

Heterobasidion parviporum Niemelä \& Korhonen (Basidiomycota; Agaricomycotina; Russulales) is a causative agent of root and butt rot disease of conifers, particularly Norway spruce (Picea abies (L.) Karst.). Together with two closely related Eurasian species (Heterobasidion annosum (Fr.) Bref. sensu stricto (s.s.) and Heterobasidion abietinum Niemelä \& Korhonen) and two North American species (Heterobasidion irregulare Garbel. \& Otrosina and Heterobasidion occidentale Otrosina \& Garbel.), it constitutes the species complex of Heterobasidion annosum sensu lato (s. 1.). The annual economic losses due to Heterobasidion infection in Europe are estimated at 800 million euros [1]. Due to its huge economic importance, most studies over the past decades have been focused on biology, ecology and control methods of Heterobasidion spp. [2]. The mechanisms underlying the pathogenesis and virulence of this species complex remain to be elucidated. This species complex infects stumps and wounds on the roots and stems through basidiospores and spreading to neighboring healthy trees by root-to-root contact [1]. It has dual lifestyles (a saprotroph feeding on wood materials and a necrotroph killing the host tissues and then feeding on dead materials) and the flexible lifestyles interchangeability makes the understanding of the fundamental pathogenesis mechanism challenging. Additionally, the lack of an efficient transformation system in Heterobasidion spp. further complicates functional verification studies [2, 3].

Presently, the complete genome sequence is only available for $H$. irregulare TC32-1 among this species complex [4]. Combining quantitative trait locus (QTL) regions with microarray data, 3 candidate genes were proposed to be accountable for the varied virulence between $H$. irregulare and $H$. occidentale [4]. By sequencing $23 \mathrm{H}$. annosum haploid isolates, a genome-wide association study (GWAS) revealed 8 virulence candidate genes located in 7 genomic regions potentially linked to the fungal growth in the sapwood of spruce and pine [5]. Genome comparison between the genotypes of $H$. irregulare and $H$. annosum indicated that pathogenesis-related genes between these two species were more conserved than genes involved in sporulation and saprotrophic decay, implying more significant roles of the latter two traits for the invasiveness of $H$. irregulare [6].

Fungal pathogen genomes driven by their hosts' evolution are remarkably plastic [7]. Genomic variations could provide insights into the evolutionary forces that shaped the genome architecture and adaptive responses of species of interest [8]. Fungal pathogens produce versatile proteins and small molecules that are subsequently secreted into extracellular spaces in response to changing environment and host conditions [9]. Components of secreted proteins and molecules are functionally relevant in breaking down extracellular carbohydrates and scavenging nutrients [10]. Moreover, phytopathogenic fungi modulate their secretomes to facilitate host colonization, to protect themselves against host-produced reactive oxygen species (ROS), and to subvert host defenses using suites of enzymes including carbohydrate-active enzymes (CAZymes), oxidoreductases, proteases and less well-generalized but often cysteine-rich, small secreted proteins (SSPs) termed effectors [11-13]. Thus, secretome could reflect fungal pathogenicity, virulence and their interactions with hosts.

The absence of $H$. parviporum reference genome results in the scarcity of data about genomic comparison at intraspecific level. Compared to its sibling species, none of the candidate genes potentially associated with its virulence have been hitherto proposed and the relationship of virulence to other fungal fitness-important phenotypic traits remains elusive. Therefore, in this study, we reported on a draft genome of one $H$. parviporum isolate and jointly analyzed this reference with 14 other re-sequenced $H$. parviporum isolates collected from different geographic locations across Finland in order to identify genomic variations that might be relevant for pathogenesis. Exploration of different classes of genes (including core genes, deleted genes, duplicated genes, reference-specific genes, novel genes relative to the reference, genes under strong selection pressure, and genes harboring nonsynonymous single nucleotide polymorphism (SNPs), nonsense mutations or frameshift mutations exclusively found in the least virulent isolate) with secreted protein coding genes more closely scrutinized in each gene class enabled us to propose some virulence candidate genes for functional analysis. Moreover, we characterized the virulence and other phenotypic traits including saprotrophic wood decay, vegetative growth and sporulation of the 15 sequenced isolates. The analysis of the correlations among different traits to virulence shed light on the traits more closely linked to the varied virulence in $H$. parviporum at phenotypic level.

\section{Methods}

Fungal isolates, single spore isolation and genotyping

All $H$. parviporum isolates of diverse geographic origins were kindly provided by Kari Korhonen (Natural Resources Institute Finland, Luke) and were maintained on $2 \%$ malt extract agar plates (MEA). Asexual conidia of each isolate were harvested by flooding the surface of plates with sterile MilliQ water and spread onto new MEA plates. After $24 \mathrm{~h}$ of incubation at $22{ }^{\circ} \mathrm{C}$, germinated conidia were picked under microscope (OLYMPUS-CX31), transferred to fresh MEA plates and incubated for 2 weeks. The hyphae of single sporederived cultures were observed under microscope for clamp connections and genotyped using 4 microsatellite markers (Ha-ms1, Ha-ms2, Ha-ms6 and Ha-ms10) as described earlier [14, 15]. Isolates that did not produce clamp connections and contained only one allele at each 
microsatellite locus analyzed were deemed as homokaryons. The verified homokaryons of each isolate used in this study were deposited at University of Helsinki Fungal Biotechnology Culture Collection (HAMBI/FBCC) (Table 1).

\section{Pathogenicity test and phenotypic characterization of fungal isolates}

Virulence assays were conducted following a previous study [16]. Briefly, Norway spruce (Picea abies) seeds (batch number: R01-00-0902-E461: courtesy of Luke) were surface sterilized with $30 \% \mathrm{H}_{2} \mathrm{O}_{2}$ for 15 min, rinsed with sterile MilliQ water and sowed on $1 \%$ water agar. Ten germinated seedlings (14-17 days old) were aseptically transferred to a $1 \%$ water agar half-covered with moist, sterile filter paper. The root regions of seedlings were inoculated with either $1 \mathrm{ml}$ of homogenized $H$. parviporum mycelia (2-3 weeks old) or sterile MilliQ water as a control and covered with a second moist, sterile filter paper. The parafilm-sealed plates were incubated at $22{ }^{\circ} \mathrm{C}$. Percentage of seedlings killed in each plate was recorded at 15 days post-inoculation (dpi) and 25 dpi with 3 plate replicates at each time point making a combined total of 30 individual seedlings per treatment. Two isolates causing the highest and the lowest mortality rate were further inoculated on 6-year-old Norway spruce clones under greenhouse condition for virulence validation based on induced necrotic lesion length in phloem and xylem (Additional file 1: Method).

Saprotrophic wood decay abilities were tested on heartwood blocks $(2 \times 1 \times 0.5 \mathrm{~cm})$ of Norway spruce according to a previous study [17]. Three wood blocks per treatment pre-dried to constant mass at $65{ }^{\circ} \mathrm{C}$ for $24 \mathrm{~h}$ were weighed, moisturized in MilliQ water for $1 \mathrm{~min}$ and placed in $100 \mathrm{ml}$ flasks containing $1 \mathrm{~g}$ of vermiculite (fraction size: $1 \mathrm{~mm}$ ) and $6 \mathrm{ml}$ of nutrient solution $\left(\mathrm{NH}_{4} \mathrm{NO}_{3} \quad 0.6 \mathrm{~g} / \mathrm{l}, \mathrm{K}_{2} \mathrm{HPO}_{4} 0.4 \mathrm{~g} / \mathrm{l}, \mathrm{KH}_{2} \mathrm{PO}_{4} 0.5 \mathrm{~g} / \mathrm{l}\right.$, $\mathrm{MgSO}_{4} .7 \mathrm{H}_{2} \mathrm{O} 0.4 \mathrm{~g} / \mathrm{l}$, glucose $\left.1.0 \mathrm{~g} / \mathrm{l}\right)$. After sealing and autoclaving, flasks were inoculated with either three agar plugs $(5 \times 5 \mathrm{~mm})$ from $H$. parviporum isolates pre-grown on Hagem media or sterile Hagem agar plugs as the control, and incubated at $22{ }^{\circ} \mathrm{C}$ for 4 months in incubation chambers with humidity maintained at $60-80 \%$. Each treatment has 5 biological replicates. Adhering mycelia were scraped off wood blocks prior to drying at $105{ }^{\circ} \mathrm{C}$ for 2 days. Percentage of dry wood mass losses with respect to original dry mass was calculated.

Vegetative growth rate was assayed by deposition of agar plugs $(5 \times 5 \mathrm{~mm})$ from growing mycelia to the center of $90-\mathrm{mm}$ Hagem agar plates. Radial growth was measured in triplicate at $7 \mathrm{dpi}$ in four perpendicular directions per plate. The average mycelia growth rates $(\mathrm{mm} / \mathrm{d})$ were calculated.

After the growth of the isolates for 2 weeks, conidia were dislodged thoroughly from four Hagem agar plugs (7 $\mathrm{mm}$ in diameter) by vigorous vortex for $2 \mathrm{~min}$ in $10 \mathrm{ml}$ of $0.1 \%$ Tween 20 solution. Conidial spore concentrations were estimated using a hemocytometer under the microscope (OLYMPUS-CX31). Each isolate has 3 replicates and sporulation was expressed as the number of conidia per milliliter.

\section{Statistical analysis}

After variance homogeneity test, differences among obtained phenotypic traits of the 15 fungal isolates were

Table 1 Summary of $H$. parviporum isolates, mapping and variants relative to the reference isolate S15

\begin{tabular}{|c|c|c|c|c|c|c|c|c|}
\hline Isolate & Name $^{a}$ & Origins & HAMBI/FBCC number & Mapped reads & Reads depth $(x)$ & Reads coverage (\%) & SNPS & InDels \\
\hline 03020 & S1 & Pernaja, Horslök & 2362 & $20,338,386$ & 47.76 & 93.52 & 146,387 & 34,661 \\
\hline 04121 & $\mathrm{~S} 2$ & Artiärvi & 2365 & $20,914,722$ & 49.13 & 92.97 & 140,039 & 34,113 \\
\hline 93242 & S3 & Siilinjärvi & 2366 & $21,352,488$ & 50.13 & 93.80 & 138,032 & 34,749 \\
\hline 04051 & S4 & Mäntsälä & 2368 & $19,057,716$ & 44.78 & 93.48 & 136,927 & 33,259 \\
\hline 99055 & S5 & Kolari, Ylläs & 2361 & $18,024,412$ & 42.34 & 93.60 & 144,819 & 34,027 \\
\hline 91271 & S6 & Karkkila & 2363 & $20,342,018$ & 47.78 & 95.21 & 94,638 & 29,362 \\
\hline 99058 & S7 & Kittilä, Kukasjärvi & 2354 & $20,662,349$ & 48.54 & 92.44 & 141,327 & 32,800 \\
\hline 01039 & S8 & Hattula, Korkee & 2369 & $18,110,965$ & 42.55 & 93.48 & 150,875 & 34,805 \\
\hline 96160 & \$9 & Askola & 2355 & $19,847,739$ & 46.58 & 93.07 & 135,994 & 32,959 \\
\hline 05029 & $\$ 10$ & Kirkkonummi, Yövilä & 2364 & $19,240,310$ & 45.20 & 93.86 & 143,212 & 33,761 \\
\hline 99067 & S11 & Loppi, Launonen & 2360 & $21,492,624$ & 50.51 & 93.13 & 143,010 & 34,019 \\
\hline 94174 & $\mathrm{~S} 12$ & Loimaa, Köyliön kylä & 2358 & $17,832,237$ & 41.90 & 92.72 & 140,559 & 32,891 \\
\hline 98038 & $\mathrm{~S} 13$ & Suomusjärvi, Kettula & 2370 & $21,039,687$ & 49.36 & 93.06 & 153,255 & 35,875 \\
\hline 03014 & S14 & Kuhmoinen & 2367 & $19,767,540$ & 46.42 & 92.79 & 143,762 & 33,717 \\
\hline 96026 & S15 & Åland Islands & 2359 & - & - & - & - & - \\
\hline
\end{tabular}


compared using analysis of variance (ANOVA) followed by Tukey HSD test. Pairwise Pearson correlation coefficients among all traits were calculated. Redundancy analysis was conducted with vegan package [18] to disentangle the contributions of phenotypic traits together with sampling sites of fungal isolates to the variation in the mortality rate of seedlings. Stepwise forward selections based on Akaike Information Criteria (AIC) were used to select the traits that best explained variation in virulence with significance assessed by 999 Monte Carlo permutation tests. Given the reduced model, variation partitioning was applied to quantify the virulence variation attributable to each selected trait, controlling for the influence of the others. All statistical analyses were performed in R v.3.0.2 [19].

\section{Genomic DNA, RNA extractions and sequencing}

Single spore-derived mycelia were harvested from 3week-old Hagem liquid cultures, grinded in sterile liquid nitrogen-cooled mortars, followed by genomic DNA isolation using DNeasy Plant Mini Kit (QIAGEN) according to manufacturer's protocol. Mycelia of the most virulent isolate selected based on virulence assay were used for total RNA extraction using TRI Reagent (Sigma Aldrich) following manufacturer's instructions. Genomic DNA and total RNA were quantified by NanoDrop spectrophotometer 2000c (Thermo Fisher Scientific Inc.) and qualities were checked via electrophoresis on a $1 \%$ agarose gel and Agilent 2100 bioanalyzer, respectively.

A paired-end ( $\mathrm{PE})$ (500-bp insert) and a mate-paired (MP) (10-Kb insert) libraries were prepared for the most virulent isolate and sequenced using an Illumina HiSeq 2500 platform (125 bp). An additional 20-Kb PacBio library was constructed and sequenced with a PacBio RSII system (Pacific Biosciences). PE libraries of 300-bp insert (90 bp) were used for remaining fungal isolates and sequenced with an Illumina HiSeq 2500 platform. RNA-seq was conducted with 100-bp PE sequencing using an Illumina HiSeq 4000 platform. All library constructions and sequencing were performed at Beijing Genomics Institute (BGI, Shen-Zhen, China).

\section{De novo genome assembly and whole genome alignment}

Reads preprocessing and de novo genome assembly of the reference isolate with a hybrid strategy were carried out at BGI. Illumina reads with adapter contaminations, or with more than $10 \%$ of ambiguous bases or with more than $40 \%$ of low quality ( $\mathrm{Q}$ score $<20$ ) bases were discarded by SOAPnuke v.1.5.2 (developed by GBI). The quality of processed reads was checked with FASTQC v. 0.11.2 (http://www.bioinformatics.babraham.ac.uk/projects/fastqc/). PacBio polymerase reads were processed by $\mathrm{SMRT}^{\oplus}$ analysis package v.2.3.0 to filter out reads with quality less than 0.8 , to remove adapters and to extract subreads with length of at least $1000 \mathrm{bp}$. The resulting subreads were self-corrected by Falcon Genome Assembly Tool Kit v.0.3.0 and assembled into contigs by Celera Assembler v.8.3 [20] followed by single base corrections using SOAPsnp v.1.05 [21] and SOAPInDel v.1.08 [22] pipelines with HiSeq PE reads. Scaffolding was performed by SSPACE- LongRead [23] using HiSeq MP reads.

Filtered reads of re-sequenced isolates were assembled de novo by VelvetOptimiser v.2.2.5 [24]. The optimized $\mathrm{k}$-mer, expected coverage and coverage cutoffs were obtained through iterative process for various k-mer (37 to 67). Gaps were minimized by GapFiller v.1.10 [25] using original reads via 15 iterations $(-\mathrm{m} 50,-\mathrm{o} 3)$.

The assembled contigs of re-sequenced isolates were aligned to the reference assembly by NUCmer in MUMmer v.3.23 [26] and filtered by delta-filter utility program to keep one-to-one best mapping of reference to query with at least $1000 \mathrm{bp}$. The alignments were visualized with dotplots generated by mummerplot and summarized by show-coords utility program in MUMmer package.

\section{Transposable elements (TEs), simple sequence repeats (SSRs), gene prediction and annotation}

TEs in reference assembly were predicted using RepeatScout v.1.0.5 [27], Nseg [28], TRF [29] and classified with REPCLASS v.1.0.1 [30] and tblastx against Repbase Update database v.29.8.2016. Full-length long terminal repeat (LTR) retrotransposons were identified by LTRharvest [31] and LTRdigest [32]. All detected classified TEs were soft-masked by RepeatMasker v.4.0.6 (http://www.repeatmasker.org/) (Additional file 2: Method).

SSRs were scanned by SciRoKo v.3.4 [33] with perfect MISA-mode (default setting). SSR motifs were standardized by grouping motifs in different reading frames and corresponding reverse complements together. The distribution of SSRs relative to protein-coding genes (CDS, introns, 200 bp up- and downstream of CDS, 200-500 bp up- and downstream of CDS and $>500$ bp up- and downstream of CDS) were inspected with BEDtools v.2.26.0 [34].

Protein-coding genes were identified using the modified fungal genome annotation pipeline as described by previous studies $[35,36]$. After removing adapter contaminations, low quality reads ( $Q$ score $<20$ ) and rRNA remnants, remaining RNA-seq reads were constructed into transcripts using both de novo and genome-guided methods (jaccard_clip option on) in Trinity v2.3.2 [37]. For the genome-guided method, RNA-seq reads were first aligned to the genome assembly by GSNAP v.2014. 7.21 [38] to generate a BAM file as the input. The resulting transcripts from these two modes were combined and aligned to the genome assembly with GMAP v.2014. 7.21 [39] incorporated in Program to Assemble Spliced Alignments (PASA) pipeline v.2.0.2 [40] to build a 
complete set of transcripts. Homologs from predicted proteins of $H$. irregulare TC 32-1 available in Mycocosm at Joint Genome Institute (JGI) portal (http://genome.jgi. doe.gov/Hetan2/Hetan2.home.html) were searched in the soft-masked assembly by Exonerate v.2.2.0 [41] with protein2genome model. GeneMark-ES v.4.21 [42] with self-training algorithm, fungal branch point model was utilized for $\mathrm{ab}$ initio gene predictions. All evidences generated above were sent into EVidenceModeler v.1.1.1 [40] to construct weighted consensus gene structures. The weights were set as 1,5 , and 10 for ab initio predictions, homology-based predictions, and transcripts, respectively. A subset of 806 complete gene models with exact exon-intron boundaries as PASA-constructed transcripts and a support from exonerate-based homologs were manually selected for training Augustus v.3.2.2 [43] and SNAP v.2013.11.29 [44]. The trained programs were used to predict genes ab initio with and without evidence from GSNAP mapping, Exonerate protein alignments and identified known repeats. Thereafter, all predicted gene models were combined into consensus gene structures by EVidenceModeler with the weights of $2,3,5$, and 7 for ab initio gene predictions without and with hints, homology-based predictions and transcripts, respectively. The complete gene set was subjected to PASA to add UTR annotations based on transcript alignments. The completeness of predicted gene set was assessed by Benchmarking Universal Single-Copy Orthologs (BUSCO) analysis using 1438 fungal single-copy ortholog profiles [45].

The predicted protein sequences were functionally annotated with BLAST2GO v.4.0.7 pipeline [46] (default value). Protein sequences were first blastp against the nonredundant protein database in NCBI (threshold Evalue $\leq 1 \mathrm{e}-5)$. Hits were used to assign Gene Ontology (GO) terms to each protein sequence. InterProScan v.5. 15-55 [47] was performed to search protein sequences against a collection of databases including Gene3D v.5.5. 0, HAMAP v.201511.02, PANTHER v.10.0, Pfam v.28.0, PIRSF v.3.01, PRINTS v.42.0, ProDom v.2006.1, ProSitePattern v.20.113, ProSiteProfiles v.20.113, SMART v.6.2, SUPERFAMILY v.1.75, TIGRFAM v.15.0 to identify present domains and motifs. Meanwhile, available corresponding GO terms for each hit were also retrieved and merged to existing GO annotations from BLAST2GO. CAZymes were annotated by dbCAN v.5.0 with HMMER3 (default setting) [48]. The whole genome was visualized with Circos v.0.69.5 [49].

\section{Secretome prediction}

Secreted proteins were identified using SignalP v.4.1 (sensitive mode) [50] for signal peptide prediction, TargetP v.1. 1 [51] for subcellular locations and TMHMM v.2.0 [52] for transmembrane (TM) domain detection. Proteins with more than two TM domains or with one TM domain not overlapping with signal peptide (helix of at least 18 amino acids not in the first 60 amino acids) were excluded. The predicted secretome was further searched in MEROPS $\mathrm{v}$. 11.0 [53] and PeroxiBase v.2004-2015 [54] with blastp (Evalue $\leq 1 \mathrm{e}-4$ ) for proteases and peroxidases, respectively. Potential virulence-related proteins were identified by blastp against pathogen-host interaction database (PHIbase) v.4.2 [55] (E-value $\leq 1 \mathrm{e}-5)$.

\section{Genome mapping, variant calling and genome-wide nucleotide polymorphism analysis}

Processed reads of re-sequenced isolates were mapped to the reference assembly by BWA-MEM v.0.7.12 [56]. Duplicates were removed by Picard MarkDuplicates v2.1.1 (http://broadinstitute.github.io/picard/). SNPs and small insertions/deletions (InDels) collectively termed variants were called by Genome Analysis Toolkit (GATK) v.3.6 [57] HaplotypeCaller and GenotypeGVCFs, followed by hard filtering with VariantFiltration (generic filter recommendations of GATK plus DP > 200.0, DP < 10.0). Nucleotides flanking identified SNPs were retrieved by BEDtools. Variants exclusive to each isolate were pinpointed by VCFtools v.0.1.15 [58]. The impacts of variants on different types of reference genomic regions (intergenic regions, exons, introns, 5'UTR, 3'UTR and exon-intron splicing sites) were predicted with SnpEff v.3.1 [59]. Additionally, the $125 \mathrm{PE}$ (500-bp insert) Illumina reads of the reference isolate were also mapped to the genome of $H$. irregulare TC $32-1$ by BWA-MEM.

The individual deduplicated BAM files of re-sequenced isolates were merged by SAMtools v.1.2 [60] to calculate genome-wide nucleotide diversity and Tajima's $D$ in nonoverlapping $5 \mathrm{~Kb}$ windows using PopBam v.3.0 (-m 10, $-\times 200,-\mathrm{q} 40)$ [61]. Based on the distribution of $D$-value, outlier windows ( $D$-value $<5$ th or $>95$ th percentile) were selected. Genes with at least $80 \%$ of their length overlapped in the outlier windows were extracted.

\section{Gene classification}

Mapping coverage of re-sequenced isolates over the reference gene models was calculated with BEDtools. Coverage breadth was expressed as percentage of nucleotides with minimum one read aligned. Genes were defined as core genes when shared by all isolates with minimum coverage breadth of $80 \%$. Remaining genes exhibited deletions including complete deletions (coverage breadth $\leq 10 \%$ ) or partial deletions (coverage breadth < $80 \%$ but $>10 \%$ ) in subset of re-sequenced isolates. Genes only present in the reference isolate were called reference-specific genes. Novel genes relative to the reference were identified by de novo assembling unmapped reads using VelvetOptimiser, predicted using referencetrained Augustus and annotated by blastp against NCBI 
non-redundant protein database (E-value $\leq 1 \mathrm{e}-5)$, BLAST2GO and InterProScan v.5.22-61.0 against Pfam v.30. 0 . Copy-number gains were analyzed with $\mathrm{CNVnator} v$. 0.3.3 [62] using deduplicated BAM files. Regions with putative copy number gains were extracted using a bin size of $80 \mathrm{bp}$ recommended by the software and filtered with $p$-value $<0.05$ and duplication level $\left(\mathrm{q}_{0}\right)<0.5$. Genes with at least $80 \%$ of their length overlapped in the copy number gained regions were considered as duplicated genes. Significant frequency differences in GO terms between selected gene sets and all gene models were performed by Fisher's exact test with false discovery rate $(F D R)<0.05$. Gene conservation was assessed by the number of variants detected above. Defined conserved genes had less than 5 variants/Kb without nonsynonymous SNPs and HIGH impact variants annotated by SnpEFF v.3.1 [59], whereas divergent genes possessed at least 40 variants/Kb.

\section{Results}

Phenotypic characterizations of homokaryotic isolates Inoculation of $15 \mathrm{H}$. parviporum homokaryotic isolates originating from diverse geographic locations across Finland (Table 1, Fig. 1) revealed significant differences in mortality rate of infected spruce seedlings at both 15 dpi $(F=8.723, P<0.001)$ and $25 \mathrm{dpi}(F=7.739, P<0$.
001). Isolate S15 was the most virulent, capable of killing all seedlings at $15 \mathrm{dpi}$, whereas isolate $\mathrm{S} 12$ was the least virulent, resulting in only $6.67 \%$ of seedlings killed at 25 dpi (Fig. 2a, Additional file 3: Figure S1). Seedlings in control remained healthy during the experimental period. Variations in wood decay capability, vegetative growth and sporulation were also observed among all isolates (Fig. 2b, Additional file 4: Figure S2 a,b). Further virulence validation of the isolates S15 and S12 in greenhouse using 6-year-old Norway spruce clones supported their drastically differed virulence as reflected by induced necrotic lesion lengths in phloem and xylem (Fig. 2c, Additional file 4: Figure S2c). However, no significant pairwise correlations between the measured traits were observed except for a moderate correlation $(r=0.54$, $P<0.05)$ between wood decay and mortality rate at 15 dpi. Amongst all measured traits together with longitude and latitude of fungal isolates' sampling sites, wood decay, latitude and an interaction between these two terms were significant factors in determining the variation in mortality rate, explaining $66.46 \%$ of the variance $(P<0.01)$ with latitude $(44.46 \%)$ being more important than wood decay $(17.56 \%)$ and their interaction term (16.72\%) (Additional file 4: Figure S2d). Interestingly, the significance of latitude depends on the coexistence of wood decay in the initial analytical model and vice versa

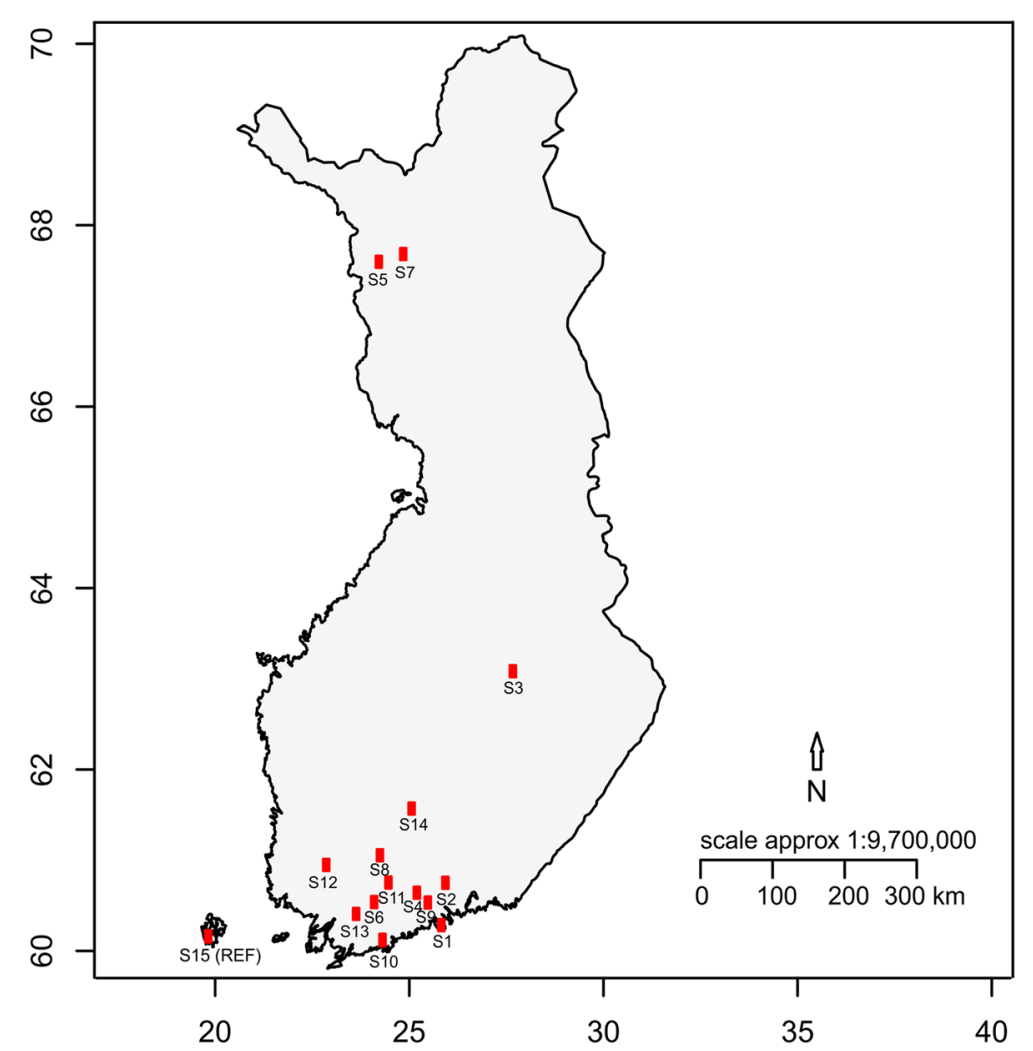

Fig. 1 Sampling sites of the $15 \mathrm{H}$. parviporum isolates used in this study in Finland. X-and $y$-axes are longitude and latitude, respectively 


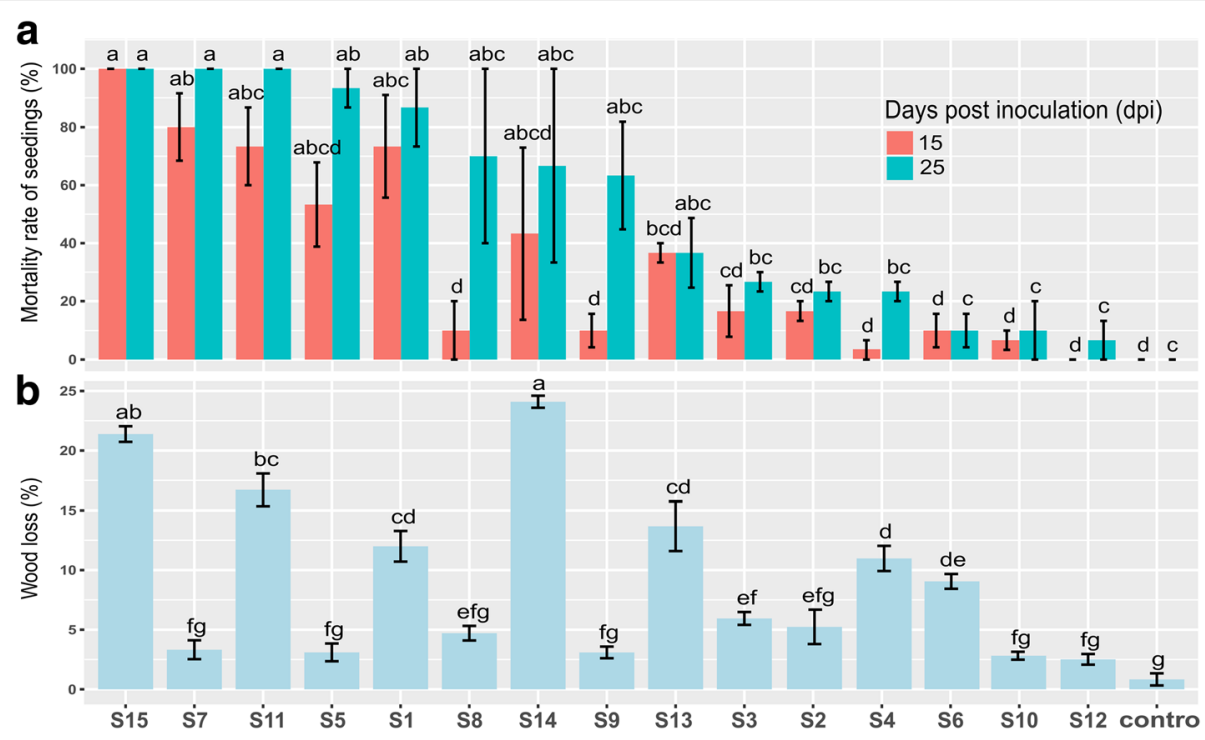

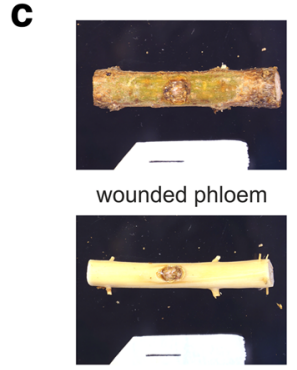

wounded xylem

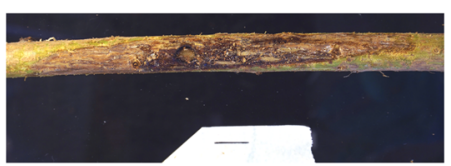

$\mathrm{S} 15$ infected phloem

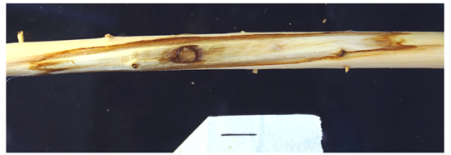

S15 infected xylem

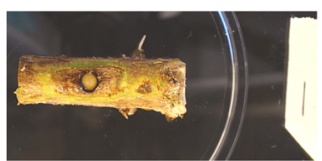

S12 infected phloem

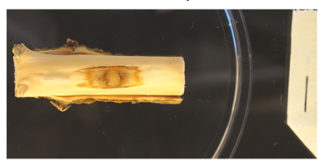

S12 infected xylem

Fig. 2 Phenotypic characterization of $15 \mathrm{H}$. parviporum isolates and virulence validation of two selected isolates. a Mortality rate of Norway spruce seedlings infected by 15 Heterobasidion parviporum isolates and the control at 15 and 25 dpi. b Percentage of wood dry mass losses caused by $15 \mathrm{H}$. parviporum isolates and the control. c Norway spruce clone C30560 infected by S15 and S12 as well as wounded control in phloem and xylem in virulence validation. Black line is $10 \mathrm{~mm}$ scale bar

despite the opposite effects these two factors have on virulence (negative variances in overlapped regions in Venn diagram) (Additional file 4: Figure S2d).

\section{Genome assembly, transposable elements (TEs), simple sequence repeats (SSRs) and annotation of S15}

A total of 30.4 million (PE library) and 15.53 million (MP library) of 125-bp filtered PE reads and 0.42 million of filtered PacBio subreads with average length of $7.3 \mathrm{~Kb}$ were assembled into $37.76 \mathrm{Mb}$, consisted of 287 scaffolds with N50 of $630.6 \mathrm{~Kb}$ and GC content of 53\% (Table 2). A total of $60.11 \%$ reads from PE library of S15 could be properly mapped onto H. irregulare TC32-1 genome, covering 66. $24 \%$ of entire $H$. irregulare genome. This was expectedly lower than $H$. annosum reads coverage on $H$. irregulare genome $(75 \%)$ as $H$. annosum and $H$. irregulare have more comparable host preference for the genus Pinus [6]. The lower genome coverage further necessitated the sequencing of $H$. parviporum reference genome to achieve higher resolution of genetic variations and to facilitate the functional study in this species.

The identified TEs occupied $20.29 \%$ of the assembly, falling into classes of non-LTR retrotransposons $(0.28 \%)$, LTR retrotransposons (14.68\%), DNA transposons (0. 92\%) and an uncategorized group (5.02\%). The most abundant elements were Ty3/gypsy retrotransposons, covering $13.36 \%(5.04 \mathrm{Mb})$ of the full genome (Additional file 5: Table S1). The genomic regions of low GC content $(<45 \%)$ were mainly occupied by TEs (Fig. 3a,b).

The total number of SSRs found was 3066, distributed in 119 scaffolds with average length of $22.90 \mathrm{bp}$. Trinucleotide SSRs were the most frequent repeat type (Additional file 6: Table S2) and CCG, AGC, ACG were the most abundant SSR motifs (Additional file 7: Table S3). When the locations of SSRs were concerned, CDS had the highest number of SSRs (1108) dominated by trinucleotides and hexanucleotides. The pronounced dominance of trinucleotides was also observed in $200 \mathrm{bp}$ and $200-500 \mathrm{bp}$ regions upstream of CDS. In contrast, tetranucleotides 
Table 2 Summary of genome assembly and annotation in isolate $\mathbf{S 1 5}$

\begin{tabular}{|c|c|}
\hline Feature & Value \\
\hline Scaffold number ${ }^{a}$ & 287 \\
\hline Total scaffold length $(\mathrm{Mb})^{\mathrm{a}}$ & 37.76 \\
\hline Scaffold N50 (Kb) & 630.6 \\
\hline Scaffold N90 (Kb) & 89.4 \\
\hline Maximum scaffold length (Mb) & 3.49 \\
\hline Minimum scaffold length (Kb) & 4.1 \\
\hline $\mathrm{GC}$ content $(\%)^{\mathrm{a}}$ & 53.00 \\
\hline Number of predicted genes ${ }^{a}$ & 10,502 \\
\hline $\begin{array}{l}\text { Number of genes with RNA-seq } \\
\text { support }^{b}\end{array}$ & 8859 \\
\hline Mean protein length (aa) & 479 \\
\hline Total gene length (Mb) & 22.35 \\
\hline Number of genes with Blast hits & 9789 \\
\hline Number of genes with InterProScan hits & 7941 \\
\hline Number of genes with GO annotations & 6977 \\
\hline $\begin{array}{l}\text { Benchmarking Universal Single-Copy } \\
\text { Orthologs (BUSCOs) assessment }{ }^{c}\end{array}$ & $\begin{array}{l}\text { C: } 92.6 \%(1332), \mathrm{D}: 11 \%(158), \\
\text { F: } 6.2 \%(89), \mathrm{M}: 1.2 \%(17)\end{array}$ \\
\hline \multicolumn{2}{|c|}{ 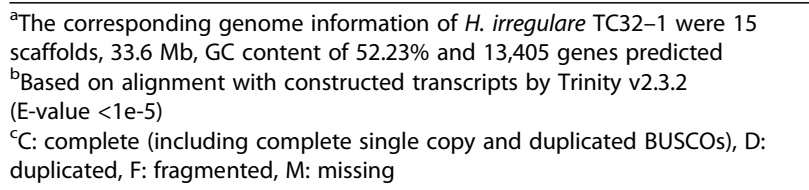 } \\
\hline
\end{tabular}

were more common in introns, regions 200 bp downstream of CDS and regions located more than $500 \mathrm{bp}$ away from CDS (Additional file 8: Figure S3).

The three classified TE classes were soft-masked in the assembly, from which 10,502 protein-coding genes were predicted and mostly concentrated on larger scaffolds (Table 2, Fig. 3c). In total, 9789 (93.2\%) and 7941 genes $(75.6 \%)$ had blast hits and InterProScan hits, respectively. GO terms could be assigned to 6977 genes (66.44\%). A number of 491 genes were categorized into CAZyme families (Additional file 9: Table S4). The BUSCO assessment showed that 1332 (92.6\%) out of 1438 nearly universal fungal single-copy orthologs were predicted full-length and 1421 (98.8\%) at least partially present with $17(1.2 \%)$ orthologs missing, indicating a high coverage of gene space (Table 2).

\section{Secretome annotation and PHI-base analysis}

In total, 759 putative secreted proteins were predicted, among which 238, 30 and 75 proteins belong to repertoires of secreted CAZymes, peroxidases, peptidases/ peptidase inhibitors, respectively (Additional files 10, 11 and 12: Figure S4, Table S5, Table S6). Closer examinations of CAZymes revealed 15, 17, 8, and 16 proteins putatively involved in degradation of plant cell wall components i.e. cellulose, hemicellulose, hemicellulose- pectin complex and pectin, respectively, whereas 43 proteins were predicted to display ligninolytic activities (Additional file 13: Table S7). Additionally, 36 proteins targeting fungal cell wall for exogenous chitin decomposition and recycling of its own cell wall including chitinases (GH18), chitin deacetylase (CE4), glucanases (GH16, GH30, GH55, GH81), and some members of other GH families such as GH27 ( $\alpha$-galactosidases) and GH92 ( $\alpha$-mannosidases) [63] were also present (Additional file 12: Table S6). In brief, S15 possesses a battery of CAZymes for both plant and fungal cell wall degradation and modification with more secreted proteins tailored to lignin degradation. Moreover, 19 proteins such as catalases, haloperoxidases, and thioredoxins required for encoding ROS-scavenging system [64] and putative secreted proteases mainly from Aspartic and Serine families were also found (Additional file 11: Table S5).

To further associate various secreted proteins to fungal virulence, pathogen-host interaction database (PHIbase), containing information on experimentally validated virulence factors in bacteria, fungi and oomycetes, was searched. In total, 211 secreted proteins have hit annotations of "reduced virulence", "loss of pathogenicity" or "effector_(plant_avirulence_determinant)", of which 133 proteins were affiliated to CAZymes, peptidases and peroxidases. In particular, aspartic peptidases (A1A), subtilisin-like serine peptidases (S8A), thioredoxins (Trx), ascorbate peroxidases (APx), class II peroxidases, chitinases (GH18) and chitin deacetylase (CE4). Almost all secreted proteins involved in cellulose and lignin degradations were also dominant in the list (Additional files 11, 12 and 13: Table S5, Table S6, Table S7) further substantiating their potential contributions to virulence. Interestingly, all predicted secreted cytochrome P450 (30 proteins) were assigned PHI-base annotations, highlighting the significance of this superfamily in pathogenesis.

\section{Whole genome comparison}

The re-sequenced isolates were assembled de novo and aligned to isolate S15 genome assembly to have a general overview of genomic similarity. The assembly size of isolate $\mathrm{S} 6$ was comparable to the reference $(39.63 \mathrm{Mb})$ but with higher degree of fragmentation and larger gaps, whereas the remaining isolates exhibited consistently smaller assembly size (32.76-33.30 Mb) (Table 3). Other properties of all isolates were generally homogenous according to the assembly metrics. After removing alignments of less than $1000 \mathrm{bp}, 91-93 \%$ of genomic sequences of re-sequenced isolates could be aligned to $\mathrm{S} 15$, amounting to $30 \mathrm{Mb}$ with at least $96 \%$ sequence identity (Table 3). Notably, S6 had relatively lower percentage of sequences participated in the alignment due to its more fragmented contigs and stringent filtering process. Nonetheless, the aligned regions showed the 


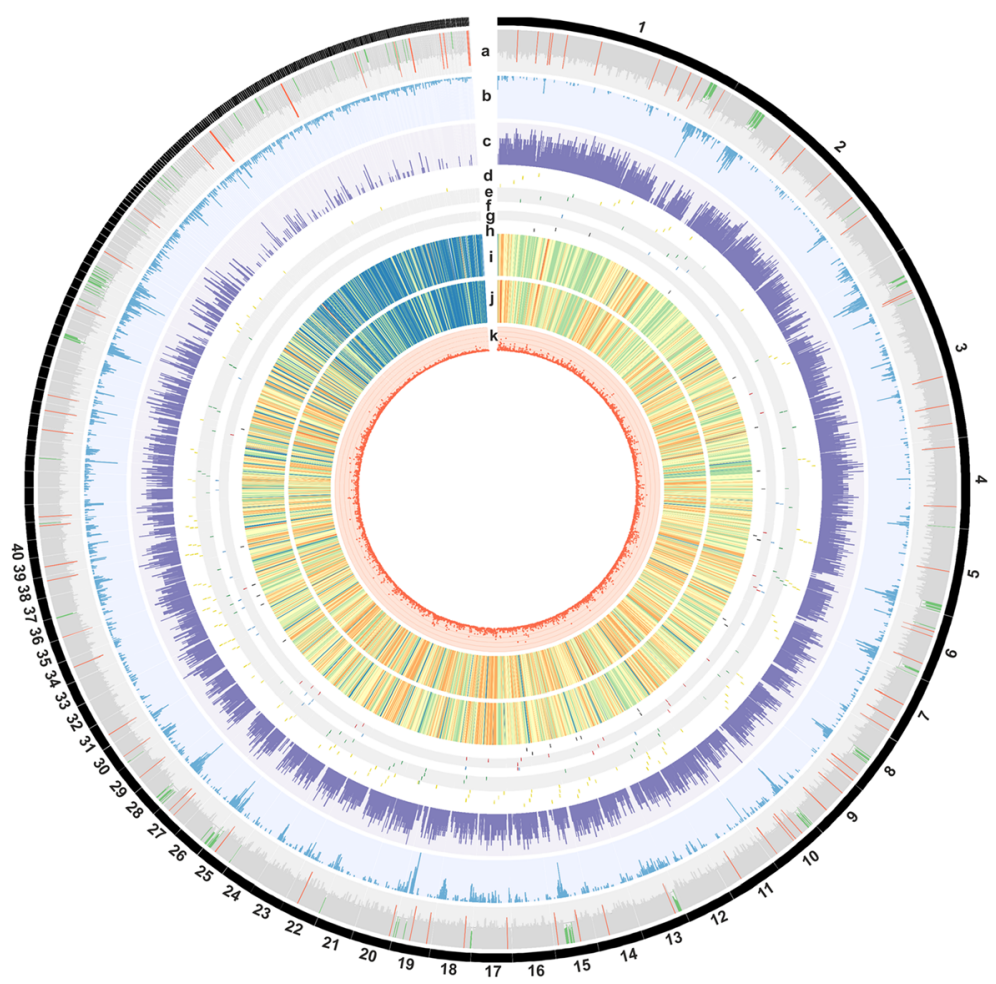

Fig. 3 The Heterobasidion parviporum S15 genome. The 287 scaffolds were arranged clockwise. The largest 40 scaffolds were labeled. Each circle from the outside to the inside depicts: (a) GC content (red > 0.65, green < 0.45); (b) Transposable elements density; (c) Gene density; (d) to (h) Secreted CAZymes, peptidase and peptidase inhibitors, peroxidases, cytochrome P450 and effector homologs of PHI-base, respectively; (i) Genome-wide nucleotide diversity, color coded from blue to red representing values from low to high; (j) SNPs density, color coded from blue to red representing values from low to high; (k) InDels density

highest nucleotide identity (Table 3). The matching scaffolds were ordered and all isolates displayed collinear configurations and overall high genomic similarity in comparison to S15 (Additional file 14: Figure S5).

\section{Variants identifications, CpG-biased mutations and genome-wide nucleotide polymorphism}

Approximately 20 million PE reads of the 14 resequenced isolates were mapped onto S15 to assess polymorphism at intra-specific level. The sequencing coverage ranged from $92.44 \%$ to $95.21 \%$ of the reference at the depth of 42 to 51 , resulting in an average of 139,488 SNPs and 33,643 InDels per isolate at 639,222 nonredundant polymorphic sites (Table 1). Among all identified variants of the 14 genomes, only $2.05 \%(10,747$ out of 524,585$)$ of SNPs and $1.07 \%$ (1354 out of 126,720 ) of InDels were shared by all isolates. Therefore, contrary to the SNP density of $3.7 / \mathrm{Kb}$ at inter-individual level, a substantially higher density was observed $(13.9 / \mathrm{Kb})$ at intra-specific level. Isolate S6 possessed markedly lower number of variants whereas isolate S13 had slightly higher number of variants. This was congruent with sequence identity in the genome alignments which showed that isolate S6 and S13 exhibited the lowest and highest sequence identity respectively (Table 1 , Table 3 ).

Most variants $(38.38 \%)$ were located in intergenic regions (Additional file 15: Figure S6). The transition to transversion ratio among all SNPs was 3.28 dominated by C-to- $\mathrm{T}(22.20 \%)$ and their complementary bases G-to-A mutations (22.23\%). Nucleotides flanking C-to-T changes showed a bias of $40.4 \%$ towards $\mathrm{G}$ at the 3 ' base (i.e. CpG-to-TpG) (Additional file 16: Table S8). As CpG-to-TpG mutation could also increase the occurrences of stop codon (TGA) in open reading frame (ORF) of protein-coding genes, stop codon usages in all predicted genes as well as secreted protein coding genes were checked. As expected, TGA displayed the highest frequency in both gene sets $(53.84 \%$ and $56.92 \%$ respectively), while TAA $(19.36 \%, 20.29 \%)$ and TAG $(26.80 \%, 22.79 \%)$ showed lower frequencies.

Nucleotide diversity variations and Tajima's $D$ value [65], a measure of the skew of allele frequency distribution across the genomes emphasize the corresponding different evolutionary potentials in different genomic regions. The genome-wide nucleotide diversity was consistent with SNP density (Fig. 3i,j). 
Table 3 Summary of assembly of re-sequenced isolates and alignments to S15 genome

\begin{tabular}{|c|c|c|c|c|c|c|c|c|}
\hline Isolates & $\begin{array}{l}\text { Contig } \\
\text { number }\end{array}$ & N50 (Kb) & $\begin{array}{l}\text { Assembly } \\
\text { size (Mb) }\end{array}$ & $\begin{array}{l}\text { GC content } \\
(\%)\end{array}$ & $\begin{array}{l}\text { N number } \\
(\%)\end{array}$ & $\begin{array}{l}\text { Aligned } \\
\text { length (Mb) }\end{array}$ & $\begin{array}{l}\text { Aligned } \\
\text { sequence (\%) }\end{array}$ & $\begin{array}{l}\text { Sequence } \\
\text { identity (\%) }\end{array}$ \\
\hline S1 & 6116 & 50.39 & 33.12 & 52.87 & 0.26 & 30.61 & 92.42 & 96.46 \\
\hline S2 & 5800 & 55.67 & 33.02 & 52.88 & 0.24 & 30.21 & 91.49 & 96.45 \\
\hline S3 & 6156 & 51.20 & 33.09 & 52.91 & 0.21 & 30.58 & 92.42 & 96.60 \\
\hline S4 & 6328 & 52.94 & 33.30 & 52.89 & 0.26 & 30.55 & 91.75 & 96.66 \\
\hline S5 & 6499 & 49.51 & 33.19 & 52.86 & 0.29 & 30.49 & 91.85 & 96.55 \\
\hline S6 & 19,952 & 44.43 & 39.63 & 52.96 & 1.01 & 30.25 & 76.33 & 97.27 \\
\hline S7 & 6016 & 45.83 & 32.93 & 52.88 & 0.22 & 30.25 & 91.87 & 96.58 \\
\hline S8 & 6096 & 47.81 & 33.02 & 52.86 & 0.23 & 30.41 & 92.09 & 96.52 \\
\hline S9 & 5838 & 52.80 & 32.95 & 52.88 & 0.21 & 30.65 & 93.01 & 96.62 \\
\hline $\mathrm{S} 10$ & 5892 & 52.53 & 33.16 & 52.89 & 0.22 & 30.68 & 92.54 & 96.69 \\
\hline S11 & 5599 & 55.60 & 32.81 & 52.92 & 0.20 & 30.40 & 92.64 & 96.59 \\
\hline $\mathrm{S} 12$ & 5883 & 53.90 & 32.76 & 52.94 & 0.21 & 30.42 & 92.85 & 96.70 \\
\hline $\mathrm{S} 13$ & 6086 & 47.96 & 33.28 & 52.86 & 0.24 & 30.39 & 91.33 & 96.24 \\
\hline S14 & 5631 & 49.64 & 32.90 & 52.85 & 0.19 & 30.21 & 91.81 & 96.43 \\
\hline
\end{tabular}

Tajima's $D$ value assessed in a 5 -Kb non-overlapping sliding window manner [66] revealed 6132 windows fulfilling the filtering criteria and the distribution of derived $D$ value showed the 5 th percentile and 95th percentile to be -1.9365 and 1.7261 respectively. As positive $D$ values represent sequences with an excess of intermediate frequency polymorphism as the result of balancing selection, whereas negative $D$ values denote the sequences with a surplus of rare alleles as the consequence of positive selection [67], genes located within positive (379 genes) and negative (393 genes) outlier windows were extracted separately (Additional file 17: Table S9). No enriched GO terms could be revealed in either gene set, probably suggesting different patterns of polymorphism were taking place in response to different classes of genes in need during adaptive evolution. As putative effectors have been shown under positive selection in other fungi $[66,68]$, secreted protein coding genes located within negative Tajima's $D$ value outlier window were scrutinized (Table 4) and could be prioritized as pathogenesis-related gene candidates for functional analysis following manual gene structure inspection.

To compare the least virulent isolate S12 more closely with the most virulent reference, secreted protein coding genes that accumulated nonsynonymous SNPs and frameshift mutations exclusively in isolate S12 were collected (Table 5). There was also a point mutation in a third nucleotide position of a codon (TAC to TAA) in one secreted protein, resulting in a premature stop codon. However, this stop gain was circumvented by mutating the original first nucleotide $\mathrm{T}$ in that particular codon to $\mathrm{C}$ as well (TAC to CAA).

\section{Gene classifications}

Reads mapping strategy was employed to have a detailed look at how genes were distributed in all isolates under study together with the polymorphisms detected above (Additional file 17: Table S9). In total, 9619 genes were shared by all isolates, whereas 863 genes presented deletions in subsets of isolates. Interestingly, 20 referencespecific genes were also found. A total of 208 genes situated in duplicated regions and from 116 to 190 novel genes were pinpointed. The secreted protein coding genes within each gene set were closely examined (Fig. 4, Additional file 18: Table S10). Isolate S6 harbored the most duplicated and novel genes and the least deleted genes, whereas isolates S3, S12 and S7 had the least duplications, the least novel genes and the most deletions, respectively (Table 6).

The reference-specific genes were mainly located in two genomic regions. Seven genes were on scaffold38 within a fragment of $22.5 \mathrm{~Kb}$, and 9 genes were on scaffold51 occupying a region of $31.4 \mathrm{~Kb}$. There were RNAseq reads providing support to the existence of those genes. Genome alignments confirmed the absence of these two regions in the 14 isolates, excluding the chances of mis-assembly of reference genome (Additional file 19: Figure S7). Five out of 7 proteins in scaffold38 had hits in PHI-base with either "reduced virulence" or "loss of pathogenicity" annotations (Table 7). On scaffold51, two secreted proteins with no functional annotation and one glucose-6-phosphate isomerase were found (Table 7). One of the secreted proteins (evm.scaffold51.36) had 187 amino acids and was cysteine-rich (20 cysteines), suggesting a likely fungal effector candidate. The remaining genes had no hits in searched databases and were not shown. 


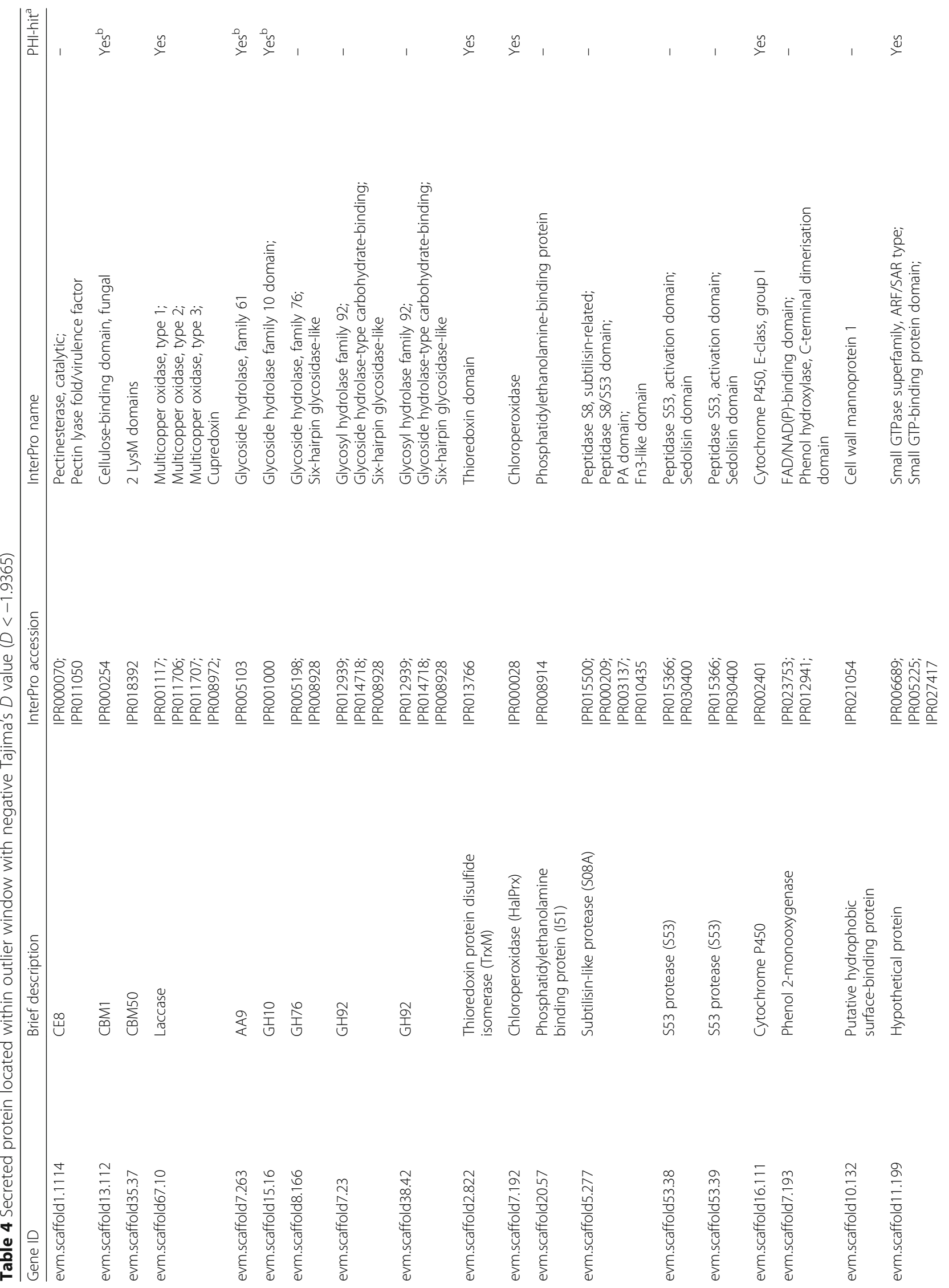


Zeng et al. BMC Genomics (2018) 19:220

Page 12 of 21

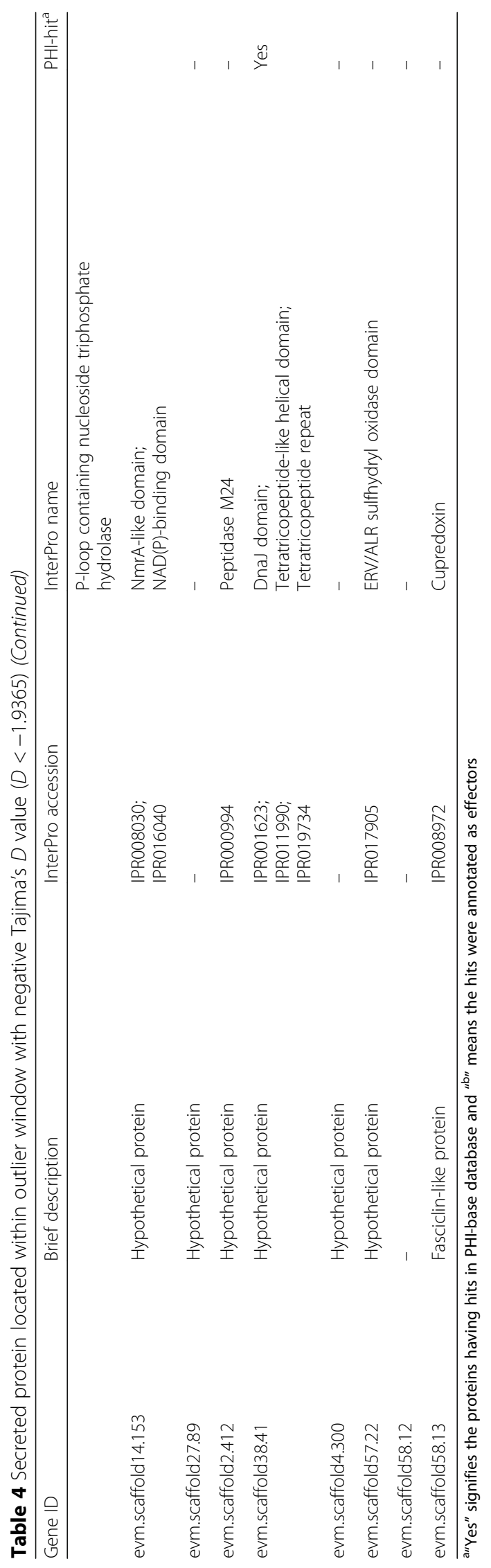


Table 5 Secreted proteins exclusively affected by nonsynonymous SNPs and frameshift mutations in S12

\begin{tabular}{|c|c|c|c|c|c|}
\hline Gene ID & Variant type & Brief description & InterPro accession & InterPro name & PHI-hit $^{a}$ \\
\hline evm.scaffold1.948 & nonsynonymous SNPs & GH18, CBM5 & $\begin{array}{l}\text { IPR003610; } \\
\text { IPR001579 }\end{array}$ & $\begin{array}{l}\text { Carbohydrate-binding module } \\
\text { family } 5 / 12 ; \\
\text { Glycoside hydrolase, chitinase } \\
\text { active site }\end{array}$ & \\
\hline evm.scaffold3.480 & nonsynonymous SNPs & PL1 & $\begin{array}{l}\text { IPR002022; } \\
\text { IPR011050 }\end{array}$ & $\begin{array}{l}\text { Pectate lyase } \\
\text { Pectin lyase fold/virulence factor }\end{array}$ & Yes \\
\hline evm.scaffold67.10 & nonsynonymous SNPs & Laccase & $\begin{array}{l}\text { IPR001117; } \\
\text { IPR011706; } \\
\text { IPR011707; } \\
\text { IPR008972; }\end{array}$ & $\begin{array}{l}\text { Multicopper oxidase, type } 1 ; \\
\text { Multicopper oxidase, type } 2 \\
\text { Multicopper oxidase, type } 3 \\
\text { Cupredoxin }\end{array}$ & Yes \\
\hline evm.scaffold31.72 & nonsynonymous SNPs & Hypothetical protein & - & - & \\
\hline evm.scaffold35.14 & nonsynonymous SNPs & Hypothetical protein & - & - & \\
\hline evm.scaffold13.87 & frameshift mutation & Hypothetical protein & - & - & \\
\hline
\end{tabular}

a"Yes" signifies the proteins cse

For the core genes, 1457 and 1456 genes fall into highly conserved and divergent gene categories, respectively. There were various significantly enriched GO terms engaged in essential biological processes in the conserved genes (Additional file 20: Table S11), in contrast to the divergent genes, which exhibited no significantly overrepresented GO terms. The most abundant GO term (biological process category) in divergent genes was oxidation-reduction process (GO:0055114) (123 genes) and the most numerous domains were relevant to transcription factors (TFs) such as Zinc finger of $\mathrm{C} 2 \mathrm{H} 2$ type, GATA type, and NF-X1 type and Zn(2)-C6

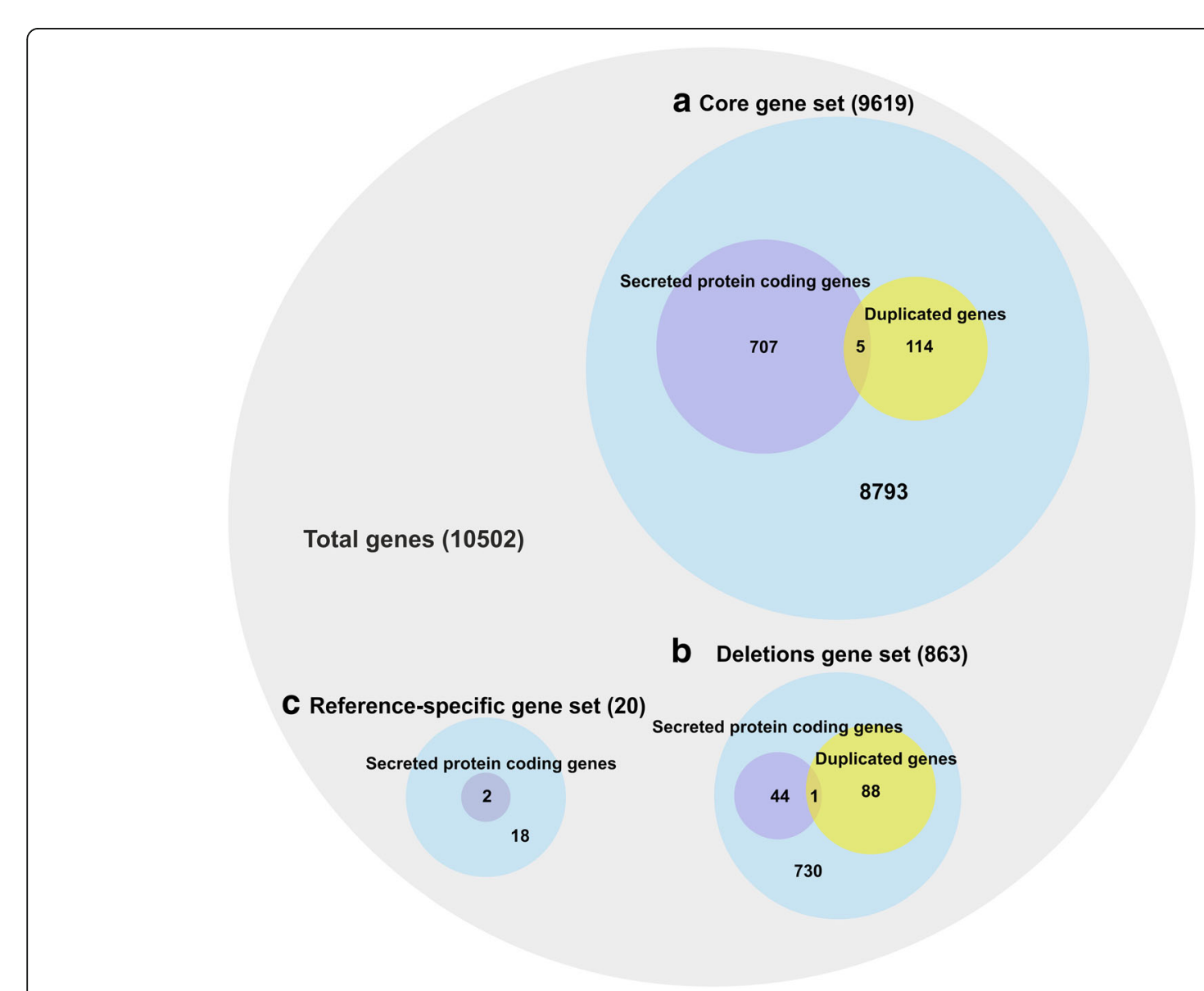

Fig. 4 Distribution of gene numbers in each gene category. Gene categories include (a) core genes, (b) deletion genes, (c) reference-specific genes. Duplicated genes and secreted protein coding genes in relation to each type of gene set were displayed 
Table 6 Summary of different categories of genes in relation to S15

\begin{tabular}{|c|c|c|c|c|c|c|c|c|}
\hline Isolates & $\begin{array}{l}\text { Duplicated } \\
\text { regions }^{a}\end{array}$ & $\begin{array}{l}\text { Duplicated } \\
\text { genes }\end{array}$ & $\begin{array}{l}\text { Completely deleted } \\
\text { genes }\end{array}$ & $\begin{array}{l}\text { Partially deleted } \\
\text { genes }\end{array}$ & $\begin{array}{l}\text { Novel } \\
\text { genes }\end{array}$ & $\begin{array}{l}\text { Novel genes } \\
\text { with Blastp hit }\end{array}$ & $\begin{array}{l}\text { Novel genes } \\
\text { with GO terms }\end{array}$ & $\begin{array}{l}\text { Novel genes } \\
\text { with domain(s) }\end{array}$ \\
\hline S1 & 62 & 34 & 91 & 212 & 142 & 114 & 39 & 34 \\
\hline S2 & 83 & 52 & 98 & 219 & 153 & 126 & 36 & 34 \\
\hline S3 & 64 & 22 & 94 & 194 & 134 & 109 & 33 & 34 \\
\hline S4 & 78 & 50 & 59 & 218 & 157 & 124 & 27 & 32 \\
\hline S5 & 67 & 50 & 82 & 195 & 156 & 137 & 35 & 39 \\
\hline S6 & 95 & 54 & 56 & 106 & 190 & 157 & 45 & 42 \\
\hline S7 & 81 & 53 & 124 & 229 & 155 & 127 & 34 & 34 \\
\hline S8 & 61 & 45 & 107 & 190 & 137 & 111 & 28 & 28 \\
\hline S9 & 49 & 28 & 95 & 219 & 130 & 104 & 23 & 26 \\
\hline $\mathrm{S} 10$ & 71 & 43 & 71 & 197 & 155 & 125 & 30 & 32 \\
\hline S11 & 58 & 32 & 103 & 213 & 121 & 99 & 24 & 24 \\
\hline $\mathrm{S} 12$ & 59 & 39 & 106 & 216 & 116 & 95 & 19 & 18 \\
\hline $\mathrm{S} 13$ & 66 & 38 & 84 & 207 & 150 & 116 & 34 & 32 \\
\hline S14 & 80 & 43 & 95 & 219 & 142 & 113 & 27 & 28 \\
\hline
\end{tabular}

${ }^{a}$ the largest and the smallest numbers in each category are in bold

fungal-type DNA-binding domain [69]. Secreted protein coding genes affiliated to the highly conserved genes (89 genes) were significantly associated with main GO terms (assigned to at least 10 sequences) of carbohydrate metabolic process (GO:0005975) and hydrolase activity, hydrolyzing O-glycosyl compounds (GO:0004553). Highly divergent secreted proteins (123 genes) displayed significant overrepresentations of main GO terms of oxidation-reduction process (GO:0055114), carbohydrate metabolic process (GO:0005975) and heme binding (GO:0020037) (Additional files 21 and 22: Table S12, Notes). Furthermore, secreted protein coding genes (21. 08 variants $/ \mathrm{kb}$ ) were slightly more polymorphic than the overall gene set in terms of variant density (19.59 variants/kb), but opposite result was found in terms of nonsynonymous SNPs density (3.46 nonsynonymous SNPs/

Table 7 Reference-specific genes on scaffold38 and scaffold51 with annotations

\begin{tabular}{|c|c|c|c|c|}
\hline Gene ID & Brief description & GO ID & $\begin{array}{l}\text { InterPro } \\
\text { accession }\end{array}$ & InterPro name \\
\hline evm.scaffold38.25 & $\begin{array}{l}\text { Short chain dehydrogenase } \\
\text { reductase }\end{array}$ & GO:0016491;GO:0055114 & $\begin{array}{l}\text { IPR002347; } \\
\text { IPR016040 }\end{array}$ & $\begin{array}{l}\text { Short-chain dehydrogenase/reductase SDR; NAD(P)- } \\
\text { binding domain }\end{array}$ \\
\hline evm.scaffold38.27 & $\begin{array}{l}\text { MFS general substrate } \\
\text { transporter }\end{array}$ & GO:0055085;GO:0016021 & $\begin{array}{l}\text { IPR011701; } \\
\text { IPR020846 }\end{array}$ & $\begin{array}{l}\text { Major facilitator superfamily; } \\
\text { Major facilitator superfamily } \\
\text { domain }\end{array}$ \\
\hline evm.scaffold38.28 & Alpha beta-hydrolase & - & IPR029058 & Alpha/Beta hydrolase fold \\
\hline evm.scaffold38.29a & Cytochrome P450 & $\begin{array}{l}\text { GO:0005506;GO:0016705; } \\
\text { GO:0055114;GO:0020037; } \\
\text { GO:0016020 }\end{array}$ & IPR001128 & Cytochrome P450 \\
\hline evm.scaffold38.31 & Transmembrane & - & $\begin{array}{l}\text { IPR008547; } \\
\text { IPR029058 }\end{array}$ & $\begin{array}{l}\text { Protein of unknown function } \\
\text { DUF829, TMEM53; } \\
\text { Alpha/Beta hydrolase fold }\end{array}$ \\
\hline evm.scaffold $38.32^{a}$ & $\begin{array}{l}\text { Short chain dehydrogenase } \\
\text { reductase }\end{array}$ & GO:0016491;GO:0008152 & $\begin{array}{l}\text { IPR002347; } \\
\text { IPR016040 }\end{array}$ & $\begin{array}{l}\text { Short-chain dehydrogenase/reductase } \\
\text { SDR; NAD(P)-binding domain }\end{array}$ \\
\hline evm.scaffold38.33 & Hypothetical protein & GO:0016020 & - & Alpha/Beta hydrolase fold \\
\hline evm.scaffold51.36 ${ }^{\mathrm{b}}$ & - & - & - & - \\
\hline evm.scaffold51.42 & $\begin{array}{l}\text { Glucose-6-phosphate } \\
\text { isomerase }\end{array}$ & $\begin{array}{l}\text { GO:0004347;GO:0006094; } \\
\text { GO:0006096; }\end{array}$ & $\begin{array}{l}\text { IPR001672; } \\
\text { IPR035476; } \\
\text { IPR035482 }\end{array}$ & $\begin{array}{l}\text { Phosphoglucose isomerase (PGI); } \\
\text { Phosphoglucose isomerase, SIS domain 1; } \\
\text { Phosphoglucose isomerase, SIS domain } 2\end{array}$ \\
\hline evm.scaffold51.44 & Hypothetical protein & - & - & - \\
\hline
\end{tabular}

a denotes the proteins having $\mathrm{PHI}$-base database hits

${ }^{\mathrm{b}}$ denotes the secreted proteins 
$\mathrm{kb}$ vs 3.84 nonsynonymous SNPs/kb). Nonetheless, the differences were not significant in either comparison (Mann Whitney test, $P=0.9803$; permutation t-test, $P=$ 0.07449 respectively).

To check the gene number variation among the resequenced isolates, a gene set with complete or partial deletions in one or more of the isolates was collected. In analogy to the divergent core genes, no significantly enriched GO terms were found. Genes encoding TFsrelated domains and those involved in oxidationreduction process (GO:0055114) were also prevalent. To link putative gene loss (coverage breath $\leq 0.1$ ) with the weak virulence of isolate S12, the 106 genes completely absent from it were checked. Isolate S7 shared 62 genes, the highest number of complete deletions with S12, despite being the second most aggressive strain on the seedlings (Fig. 2a). There was no single gene that was solely lost in S12 or in the less virulent isolate group S10, S12 and S6. Only 45 secreted proteins (6\% of secretome) had deletions. Four secreted proteins i.e. a multicopper oxidase (evm.scaffold14.101), a cytochrome P450 (evm.scaffold10.169), an acid protease (evm.scaffold35.1) and a hypothetic protein (evm.scaffold10.90) had PHI-base hits, putatively contributing to fungal virulence. Interestingly, S12 had partial deletions in 3 of them, whereas isolate S6 being less virulent than most of isolates harbored all of these 4 proteins (Fig. 5).

The 208 genes located within duplicated regions presented GO enrichment of DNA integration (GO: 0015074) (Additional file 21: Table S12). Comparatively larger portion of genes (16 genes) encoded TF-related domains. Three members from cytochrome P450 (evm. scaffold6.188, evm.scaffold6.189, and evm.scaffold18.20) were also found duplicated in one or more of isolates with one (evm.scaffold6.188) to be secreted. There were other 4 secreted proteins with each putatively duplicated in a single isolate in the core genes (Additional file 22: Notes).

The last group of genes were predicted from unmapped reads and may acquire isolate-specific novel functions. Most predicted genes encoded hypothetical proteins and the most prevalent GO term was nucleic acid binding (GO:0003676) (73 genes) followed by metal ion binding (GO:0046872) and oxidoreductase activity (GO:0016491). The yielded Pfam domains (Fig. 6) included several types of TE-related domains such as reverse transcriptase, integrase and transposases and those involved in gene expression regulations such as zinc knuckle and homeobox $\mathrm{KN}$ domain. Additional copies of cytochrome $\mathrm{P} 450$ were also found in subset of isolates. A total of 20 secreted proteins were predicted with no hits in searched databases.

\section{Discussion}

Inoculation of homokaryotic $H$. parviporum isolates on Norway spruce seedlings further confirmed that heterokaryosis is not absolutely required for the pathogenicity of $H$. parviporum homokaryons as previously observed [70, 71]. We then examined other phenotypic traits i.e. saprotrophic wood decay, vegetative growth and sporulation important for fungal fitness in $H$. parviporum. No significant correlations could be established between either sporulation or vegetative growth and fungal virulence or wood decay, which might be

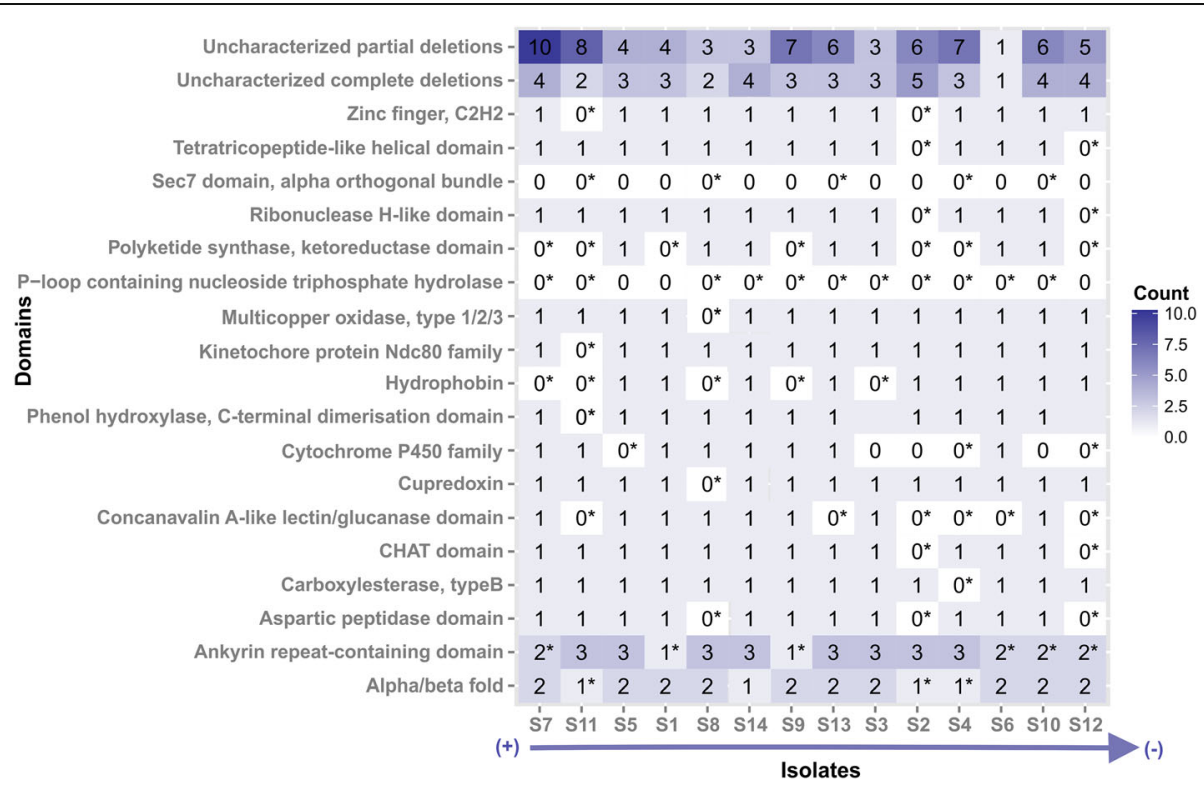

Fig. 5 Domain distribution of secreted proteins in the deletion gene set. Symbol "*" signifies the presence of partial deletions but not counted as present in the isolates. Isolates were ranked by their virulence in descending order as in Fig. 2a 


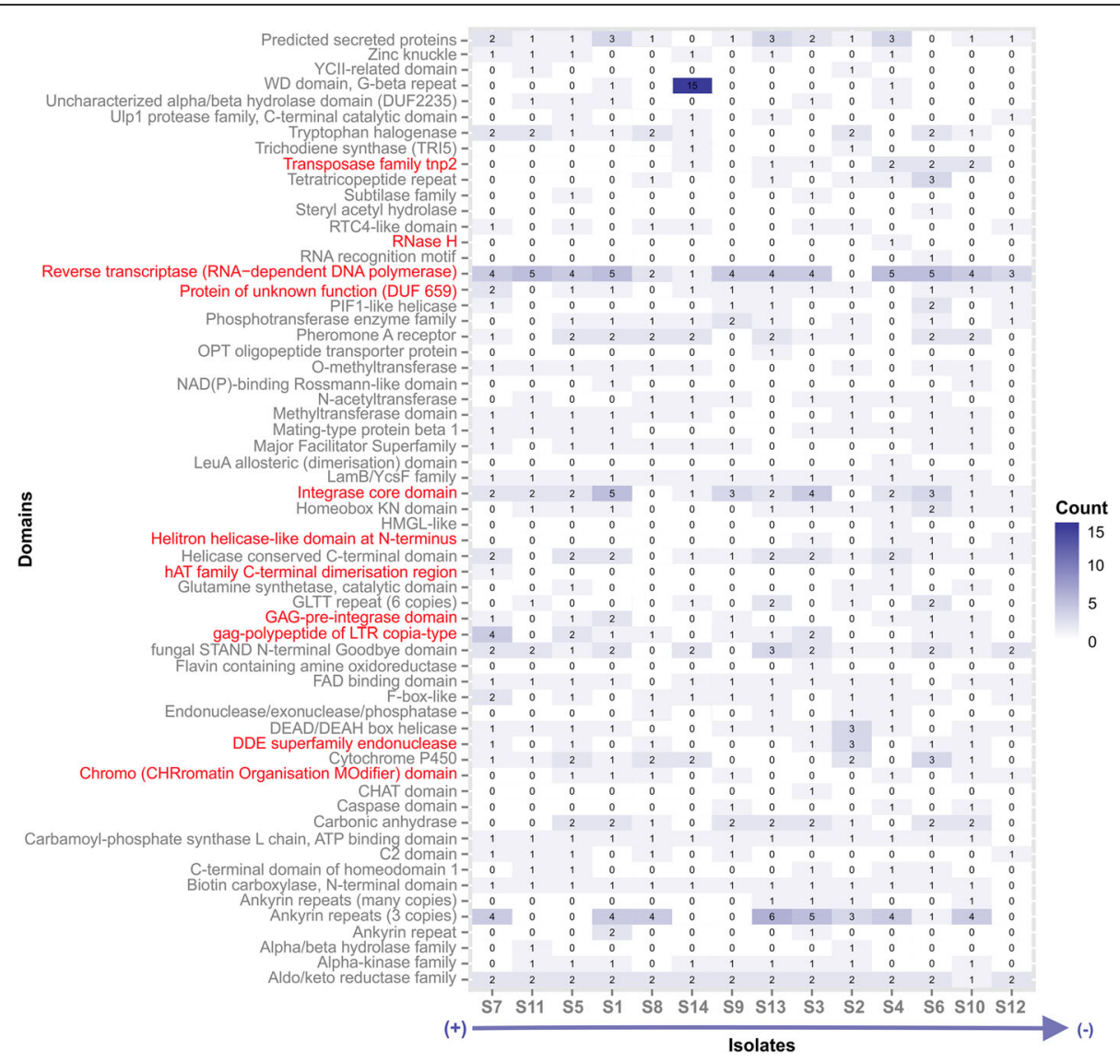

Fig. 6 Domain distribution of predicted proteins in the novel gene set. PFAM domains related to TE were highlighted in red. Isolates were ranked by their virulence in descending order as in Fig. 2a

attributed to the different fungal growth conditions. The use of seedlings in the virulence assay or wood blocks in the decay test might cause isolates to respond differently when compared to axenic culture, where vegetative growth and sporulation were assessed. For example, isolate S15 grew noticeably denser than S12 when infecting seedlings (Additional file 3: Figure S1), illustrating its superiority in colonizing hosts and aggressiveness despite its smaller mycelial growth rate on axenic culture plates. As a root pathogen of coniferous forests, latitude of fungal origins and other environmental factors such as temperature, humidity and soil type might influence the adaptability and aggressiveness of $H$. parviporum isolates. These aspects combined with their different wood decay capability might further affect the fungal virulence. However, this merits further investigation using more number of isolates from wider range of latitude. $H$. parviporum is known to switch between lifestyles (necrotroph versus saprotroph) in response to given environmental conditions. Thriving in one mode (e.g. stronger saprotrophic wood decay ability) might also better guarantee the vitality in the other mode (e.g. higher virulence).
Subsequently, all isolates were subjected to whole genome sequencing to unravel the intra-specific diversity and to explore possible molecular reason(s) underlying the variation in fungal virulence. Repetitive elements have demonstrated roles in genome shaping and adaptive evolution [72]. The observation that Ty3/Gypsy are prevailing in $H$. parviporum is in agreement to the to the pattern documented in most published fungal genomes [72]. For SSRs, a pronounced dominance of trinucleotides and hexanucleotides over non-triplet repeats in coding sequences of S15 suggested constraint selections against possible lethal frameshift mutations [73]. The dominances of trinucleotides were also observed in regions $200 \mathrm{bp}$ - and 200500 bp upstream of CDS, where putative regulatory elements were located, but attenuated with increasing distance to CDS, a similar pattern found in $H$. irregulare [4, 74]. This may reflect a significant role of trinucleotides in the regulation of gene expression with regions closer to CDS more favored by triplet selection process. Furthermore, the overrepresentation of SSRs in genes enriched in GO terms of transcription factor and regulation of transcription in $H$. irregulare provided yet another evidence of their regulatory functions. Similar to what was observed in $H$. irregulare, 
tetranucleotides were highly frequent in introns and regions 500 bp away from CDS, indicating less selection over regions more tolerant to reading frame changes. Trinucleotides CCG, AGC and ACG were abundant as similarly observed in H. irregulare, Ustilago maydis, and Fusarium graminearum $[4,75]$, suggesting that the processes of generation and fixation of those motifs were not neutral under certain yet to be characterized conditions.

The reads of re-sequenced isolates were both de novo assembled and mapped to the reference genome to inspect the genetic variations at different scales. More than 91\% of genomic sequences (except for isolate S6) could be aligned to the reference with at least $96 \%$ nucleotide identity across the whole genome. This highlighted an overall high genomic similarity within the $H$. parviporum species, which were further supported by $92-95 \%$ of genome coverage by reads. On the contrary, a striking difference of SNP density between inter-individual level $(3.7 / \mathrm{Kb}$, when each of isolate was compared to the reference) and intra-specific level $(13.9 / \mathrm{Kb}$, when all isolates were considered) reflected a substantial level of polymorphisms. The SNP density of $13.9 / \mathrm{Kb}$ in this study was higher than that within its sister taxa $H$. irregulare (4 SNPs/Kb), but lower than that of inter-specific comparison between $H$. irregulare and $H$. annosum (20 $\mathrm{SNPs} / \mathrm{Kb}$ ) [6]. Isolates in this study were collected from Finnish forests of diverse geographic origins, especially with the reference isolate from the secluded Åland Island, which may explain the higher level of polymorphisms than that of $H$. irregulare, which were all from Castelfusano Pinewood Urban Park, Rome [6].

As documented in several studies, high transition to transversion ratio with predominant C-to-T and G-to-A changes might be attributed to repeat-induced point mutation (RIP)-like activities. RIP has been experimentally identified mostly in Ascomycota species such as Neurospora crassa [76], Podospora anserina [77] and Leptosphaeria maculans [78] as one way of fungal-specific genome defense against TEs expansion. Moreover, in the phytopathogen L. maculans, RIP could act as a potential mechanism driving the diversification of effector-like genes to boost virulence [79]. In the nematode-trapping Ascomycota fungus Monacrosporium haptotylum, RIP has been additionally proposed to enable the generation of novel small-secreted proteins from long secreted proteins via introduction of premature stop codons [80]. $N$. crassa and L. maculans had CpA dinucleotide preference at C-to-T mutation sites in TEs [81]. In Basidiomycota, Microbotryum violaceum, Puccinia graminis, Melampsora larici-populina and Rhizoctonia solani have displayed similar $\mathrm{CpG}$ dinucleotide signature as $H$. parviporum presented in this study [81, 82]. However, it remains undetermined whether RIP could target CpG dinucleotides. Alternatively, CpG dinucleotide context preferred by $\mathrm{C}$-to- $\mathrm{T}$ changes could have resulted from methylation of cytosine that targeted $\mathrm{CpG}$ motifs as a part of epigenetic regulation of gene expression followed by deamination of methylcytosine that is naturally occurring in genomes [83]. Further analysis of C-to-T mutation sites across repetitive elements and protein coding regions in $H$. parviporum is needed to shed more light on the underlying process for this pattern of mutation.

Two reference-specific genomic regions with potential contributions to the fungal virulence were identified. The first region was located in scaffold38, harboring 5 out of 7 genes with PHI-base hits, while the second region was situated in scaffold51 and contained 2 secreted proteins with one of them considered as likely effector candidate. In the divergent core genes, the absence of significantly enriched GO terms suggested that genetic variations have occurred in genes of diverse functions, prevailed by oxidoreductase activity, a similar result when $H$. annosum and $H$. irregulare were compared [6]. Oxidoreductases are implicated in versatile reactions. In our study, the divergent gene family could be exemplified by cytochrome P450. Fungal P450 family has a broad range of functions from the housekeeping process of sterol biosynthesis to the more specialized functions of fungal toxins biosynthesis and xenobiotics detoxification [84, 85]. The diversified functions of this family could be manifested by the overall low sequence similarity except for the conserved domains such as the heme-binding domain or achieved by gene duplication which provides raw evolutionary genetic materials for gene family proliferation and functional diversification [86]. Gene loss is another mechanism to further modulate the P450 family size [85]. In our study, events of P450 gene duplications and gene losses in $H$. parviporum have been documented in their respective gene sets. Additional P450 were further predicted in the novel genes originated from unmapped reads, probably because of their losses from the reference isolate as well. Despite the P450 gene number variation within $H$. parviporum species, no direct relationship to the virulence could be deciphered except for one secreted P450 deleted in the subset of isolates characterized as weak virulence (Fig. 5). In light of these new data, specific classification and function assignments of $\mathrm{P} 450$ for $H$. parviporum virulence is needed.

The lack of GO enrichment in the group of genes characterized by deletions indicated that this group included genes with miscellaneous functions. Nonetheless, genes associated with oxidation-reduction process might be more prone to accumulating mutations as well as enduring gene losses as suggested by the abundance of relevant GO terms in both divergent and deletion gene sets. Efforts were then directed to link any particular complete gene deletion in isolate S12 to its weak virulence. Not a single gene was uniquely lost in S12 or in the weak virulence isolate group (S12, S10 and S6) 
compared to the reference isolate. Intriguingly, isolate S6 exhibited the highest number of genes amongst all isolates, whereas isolate S7 being the second most virulent isolate had the highest number of deletions. Hierarchical clustering of re-sequenced isolates regardless of input data type collected from the deletion set (presence/absence of all genes, GO term counts or secreted proteins domains) could not reveal clustering patterns resembling their virulence profile (data not shown). Due to the redundancy of functions in many gene families such as those common in cell wall degradation [87], it is difficult to find a direct correlation between a particular gene deletion and the fungal virulence. Taken together, it seems most probable that gene loss may not be the main driver for virulence changes.

A remarkable finding across all gene sets was the preponderance of genes encoding transcription factor (TF)related domains. TFs are crucial players in orchestrating expression of various genes involved in signal transduction [88] and are evolutionarily labile, thereby contributing to phenotypic evolution [89]. In H. parviporum, the prevalence of genes encoding TF-related domains in all featured gene sets (divergent, deletion, duplication and novel genes) further reinforced the diversity of evolutionary scenarios that TFs and their associated regulatory networks could possibly be implicated in. For example, $\mathrm{C} 2 \mathrm{H} 2$ type of zinc finger protein was found duplicated in almost all isolates, which has been considered as one way of linagespecific expansion for adaptation in vertebrates [88]. It has been documented that deleting genes encoding a secreted superoxide dismutase and catalases could not affect the virulence in the biotrophic pathogen Claviceps purpurea, whereas the deletion of a specific TF, ATF1 that functions as a general regulator of catalase activity resulted in an oxidative burst-like reaction in plant tissues and reduced fungal virulence on rye $[90,91]$. Since the gene number and the $H$. parviporum virulence were not linear dependent, it is likely that the regulatory networks might be more important than other mechanisms in affecting the fungal virulence by balancing the gene number and robustness of their responses during conifer infections. These were also probably modulated by involvements of mentioned trinucleotides upstream CDS regions or CpGbiased mutation. Therefore, gene expression profiling at specific stages during the host-pathogen interactions may help illuminate the precise role of specific genes. Technically, automated gene annotation may be prone to mistakes and gene number estimates by reads mapping have to be taken with discretion. However, our study was not aimed to obtain the precise gene number of different gene families in all isolates but rather to compare different classes of genes predicted by using the same set of criteria.

Finally, secretome in $H$. parviporum being more polymorphic than the overall gene set but with smaller nonsynonymous SNPs density may reflect the stronger positive selection that are undergoing in the secretome to remove deleterious variants in coding sequences. Therefore, secreted protein coding genes located within outlier window of negative $D$ value could be considered as good candidates for future functional analysis.

\section{Conclusions}

We reported on the first reference genome sequence for the Norway spruce pathogen Heterobasidion parviporum. Intraspecific comparative genomics analysis revealed the remarkable level of polymorphism in this species with a bias for CpG to TpG mutations, which opens up possibilities of different molecular mechanisms to be uncovered in Heterobasidion spp. Two genomic regions exclusively found in the most virulent reference isolate might contribute to its higher virulence. Genes involved in oxidation-reduction process and encoding transcription factor related domains were found to display copy number variation and nucleotide polymorphism in the studied $H$. parviporum isolates. However, not a single gene could be pinpointed as a sole determinant for their varied virulence in this fungal species. This data, therefore, urges the use of RNA-seq to compare the robustness of specific genes during the host infection process. Finally, several secreted protein coding genes proposed based on selection pressure or featured variants could prioritize the selection of putative virulence candidates for further functional analysis.

\section{Additional files}

Additional file 1: Methods. Virulence validation of two selected isolates in greenhouse. (DOCX $12 \mathrm{~kb}$ )

Additional file 2: Methods. Transposable elements identification. (DOCX $18 \mathrm{~kb}$ )

Additional file 3: Figure S1. Norway spruce seedlings infected by isolate S15 (upper row) and S12 (lower row) at $15 \mathrm{dpi}$ and $25 \mathrm{dpi}$ respectively in virulence assay. (TIFF $2918 \mathrm{~kb}$ )

Additional file 4: Figure S2. (a) Sporulation and (b) Vegetative growth rate of $15 \mathrm{H}$. parviporum isolates. Isolates were sorted by mortality rate at $25 \mathrm{dpi}$ in descending order. (c) Lesion lengths in phloem and xylem caused by isolate S12, isolate S15 and wounding treatments in virulence validation. Different lowercase letters indicate statistically significant differences $(P<0.001)$. Error bars stand for standard errors. (d) Variation partitioned by wood decay, latitude and their interaction term. (TIFF $1303 \mathrm{~kb})$

Additional file 5: Table S1. Summary of transposable elements in S15. (DOCX $14 \mathrm{~kb}$ )

Additional file 6: Table S2. Summary of simple sequence repeats (SSRs) in S15. (DOCX $12 \mathrm{~kb}$ )

Additional file 7: Table S3. The most frequent fully standardized SSR motifs in S15. (DOCX $12 \mathrm{~kb}$ )

Additional file 8: Figure S3 Simple sequence repeats (SSRs) distribution in selected genomic regions of S15. Numbers on the bars represent the total SSRs number in that particular type of region. (TIFF $321 \mathrm{~kb}$ ) 
Additional file 9: Table S4. Summary of CAZymes in S15 proteome. (DOCX $17 \mathrm{~kb}$ )

Additional file 10: Figure S4 Identified families of peptidase and peptidase inhibitors, peroxidases and CAZymes in isolate S15 secretome. (TIFF 1667 kb)

Additional file 11: Table S5. Summary of peptidases, peptidase inhibitors and peroxidases in S15 secretome. (DOCX 14 kb)

Additional file 12: Table S6. Summary of CAZymes in S15 secretome. (DOCX $15 \mathrm{~kb}$ )

Additional file 13: Table S7. Repertoire of CAZymes targeting plant cell walls (PCW) and lignin in S15 secretome. (DOCX $14 \mathrm{~kb}$ )

Additional file 14: Figure S5. Genome alignment between $H$. parviporum isolate S15 scaffolds and the contigs of re-sequenced isolates. The dot plot represents one to one best mapping, and dots on the diagonal denote co-linearity between the two genomes. Red dots stand for matches in the forward direction and blue dots are inversions relative to S15 scaffolds. (TIFF $2132 \mathrm{~kb}$ )

Additional file 15: Figure S6. Variant distributions in different type of genomic regions. (TIFF $211 \mathrm{~kb}$ )

Additional file 16: Table S8. Nucleotides counts and their frequencies within 1 bp flanking C-to-T mutations. (DOCX $13 \mathrm{~kb}$ )

Additional file 17: Table S9. Annotation of genes in different categories. (a) core genes (b) deleted genes (c) duplicated genes (d) GO terms in novel genes (e) Pfam domains in novel genes (e) genes in negative tajima's $D$ extreme window $(E W)(f)$ genes in positive tajima's $D$ EW. (XLSX 2646 kb)

Additional file 18: Table S10. Annotation of secretome in S15 (including all database searches and gene classification results). (XLSX $186 \mathrm{~kb})$

Additional file 19: Figure S7. Dot plots of alignments of S12 contigs to the corresponding regions of reference (a) scaffold38 and (b) scaffold51. (TIFF $301 \mathrm{~kb}$ )

Additional file 20: Table S11. Significantly over-represented GO terms of conserved core genes compared with all genes in S15. (DOCX $19 \mathrm{~kb}$ )

Additional file 21: Table S12. Significantly over-represented GO terms of selected test gene sets against all genes in S15. (DOCX $15 \mathrm{~kb}$ )

Additional file 22: Notes. Divergent, conserved and duplicated secreted protein coding genes. (DOCX $24 \mathrm{~kb}$ )

\section{Abbreviations}

AA: Auxiliary activities; AIC: Akaike Information Criteria; ANOVA: Analysis of variance; APx: Ascorbate peroxidases; BUSCOs: Benchmarking Universal Single-Copy Orthologs; CAZyme: Carbohydrate-active enzyme;

CBM: Carbohydrate-binding module; CE: Carbohydrate esterase; DNA: Deoxyribonucleic acid; Dpi: Days post-inoculation; FDR: False discovery rate; GH: Glycoside hydrolase; GO: Gene ontology; GT: Glycosyltransferase; GWAS: Genome-wide association study; InDel: Insertion/deletion; LTR: Long terminal repeat; MEA: Malt extract agar; MP: Mate-paired; PE: Paired-end; PHIbase: Pathogen-host interaction database; PL: Polysaccharide lyase; QTL: Quantitative trait locus; RNA: Ribonucleic acid; ROS: reactive oxygen species; SNP: Single nucleotide polymorphism; SSP: Small secreted protein; SSR: Simple sequence repeat; TE: Transposable element; TF: Transcription factor; TM: Transmembrane; Trx: Thioredoxin

\section{Acknowledgements}

We are grateful to CSC (IT Center for Science, Finland) for computational resources. We are also grateful to Kari Korhonen for providing the origina heterokaryotic Heterobasidion isolates. We equally thank Dr. Francis Martin for facilitating the collaboration with his research group and Juha Puranen (Luke) for laboratory assistance.

\section{Funding}

This project was financially supported by Academy of Finland research grant (grant number 276862). ZZ was supported by doctoral program in sustainable use of renewable natural resources (AGFOREE) at the University of Helsinki. The funders had no role in study design, data collection and analysis, decision to publish, or preparation of the manuscript.

\section{Availability of data and materials}

The datasets supporting the conclusion of this article are included within the article and its additional files, as well as in the NCBI SRA (https:// www.ncbi.nlm.nih.gov/sra/) under Bioproject PRJNA400846 (https:// www.ncbi.nlm.nih.gov/bioproject/) and SRP116620. The Whole Genome Shotgun project of the $H$. parviporum 96026 has been deposited at DDBJ/ ENA/GenBank (https://www.ncbi.nlm.nih.gov/genome/) under the accession PDUQ00000000. The version described in this paper is version PDUQ01000000

\section{Authors' contributions}

FOA conceived the study; FOA and ZZ designed the experiment and interpreted the data; $Z Z$ performed the experiment, analyzed the data and wrote the manuscript; HS conducted the single spore isolation and generated the homokaryotic isolates; EJV genotyped and validated the homokaryotic isolates; AK and TR interpreted the data; SD and EM contributed to the data analysis; FOA, AK, SD, TR, HS and EJV edited and revised the manuscript. All authors read and approved the final manuscript.

\section{Ethics approval and consent to participate}

Not applicable.

\section{Consent for publication}

Not applicable.

\section{Competing interests}

The authors declare that they have no competing interests.

\section{Publisher's Note}

Springer Nature remains neutral with regard to jurisdictional claims in published maps and institutional affiliations.

\section{Author details}

${ }^{1}$ Department of Forest Sciences, University of Helsinki, Helsinki, Finland. ${ }^{2}$ Collaborative Innovation Center of Sustainable Forestry in Southern China, College of Forestry, Nanjing Forestry University, Nanjing, China. ${ }^{3}$ Natural Resources Institute Finland (Luke), Helsinki, Finland. ${ }^{4}$ INRA UMR 1136 Interactions Arbres Micro-organismes, INRA Centre Grand Est Nancy, Champenoux, France. ${ }^{5}$ UMR 1136 Interactions Arbres/Microorganismes, Faculté des Sciences et Technologies, Université de Lorraine, Vandoeuvre-lès-Nancy, France.

Received: 7 November 2017 Accepted: 20 March 2018 Published online: 27 March 2018

References

1. Asiegbu FO, Adomas A, Stenlid J. Conifer root and butt rot caused by Heterobasidion annosum (Fr.) Bref. S.L. Mol Plant Pathol. 2005;6(4):395-409.

2. Garbelotto M, Gonthier P. Biology, epidemiology, and control of Heterobasidion species worldwide. Annu Rev Phytopathol. 2013;51:39-59.

3. Lind M, Stenlid J, Olson A. Heterobasidion annosum S.L. genomics. Adv Bot Res. 2014:70:371-96.

4. Olson A, Aerts A, Asiegbu F, Belbahri L, Bouzid O, Broberg A, Canback B, Coutinho PM, Cullen D, Dalman K, et al. Insight into trade-off between wood decay and parasitism from the genome of a fungal forest pathogen. New Phytol. 2012:194(4):1001-13.

5. Dalman K, Himmelstrand K, Olson A, Lind M, Brandstrom-Durling M, Stenlid J. A genome-wide association study identifies genomic regions for virulence in the non-model organism Heterobasidion annosum s.S. PLoS One. 2013; 8(1):e53525.

6. Sillo F, Garbelotto M, Friedman M, Gonthier P. Comparative genomics of sibling fungal pathogenic taxa identifies adaptive evolution without divergence in pathogenicity genes or genomic structure. Genome Biol Evol. 2015;7(12):3190-206

7. Stukenbrock EH, Croll D. The evolving fungal genome. Fungal Biol Rev. 2014;28(1):1-12

8. Raffaele $S$, Kamoun S. Genome evolution in filamentous plant pathogens: why bigger can be better. Nat Rev Microbiol. 2012;10(6):417-30. 
9. Girard V, Dieryckx C, Job C, Job D. Secretomes: the fungal strike force. Proteomics. 2013;13(3-4):597-608.

10. McCotter SW, Horianopoulos LC, Kronstad JW. Regulation of the fungal secretome. Curr Genet. 2016;62(3):533-45.

11. Kim KT, Jeon J, Choi J, Cheong K, Song H, Choi G, Kang S, Lee YH. Kingdom-wide analysis of fungal small secreted proteins (SSPS) reveals their potential role in host association. Front Plant Sci. 2016;7:186.

12. Persoons A, Morin E, Delaruelle C, Payen T, Halkett F, Frey P, De Mita S, Duplessis S. Patterns of genomic variation in the poplar rust fungus Melampsora lariciPopulina identify pathogenesis-related factors. Front Plant Sci. 2014:5:450.

13. Sperschneider J, Dodds PN, Taylor JM, Duplessis S. Computational methods for predicting effectors in rust pathogens. Methods Mol Biol. 2017;1659:73-83.

14. Johannesson $\mathrm{H}$, Stenlid J. Nuclear reassortment between vegetative mycelia in natural populations of the basidiomycete Heterobasidion annosum. Fungal Genet Biol. 2004;41(5):563-70.

15. Vainio EJ, Hantula J. Taxonomy, biogeography and importance of Heterobasidion viruses. Virus Res. 2016;219:2-10.

16. Asiegbu FO, Johansson M, Stenlid J. Reactions of Pinus sylvestris (scots pine) root tissues to the presence of mutualistic, saprotrophic and necrotrophic micro-organisms. J Phytopathol. 1999;147(5):257-64.

17. Oghenekaro AO, Daniel G, Asiegbu F. The saprotrophic wood-degrading abilities of Rigidoporus microporus. Silva Fennica. 2015;49(4):1-10.

18. Dixon P. VEGAN, a package of $R$ functions for community ecology. J Veg Sci. 2003;14(6):927-30.

19. R-Core-Team: R: a language and environment for statistical computing 2013.

20. Berlin K, Koren S, Chin CS, Drake JP, Landolin JM, Phillippy AM. Assembling large genomes with single-molecule sequencing and locality-sensitive hashing. Nat Biotechnol. 2015;33(6):623-30.

21. Li RQ, Li YR, Fang XD, Yang HM, Wang J, Kristiansen $K$, Wang J. SNP detection for massively parallel whole-genome resequencing. Genome Res. 2009;19(6):1124-32.

22. Li ST, Li RQ, Li H, Lu JL, Li YR, Bolund L, Schierup MH, Wang J. SOAPindel: efficient identification of indels from short paired reads. Genome Res. 2013; 23(1):195-200.

23. Boetzer M, Pirovano W. SSPACE-LongRead: scaffolding bacterial draft genomes using long read sequence information. Bmc Bioinformatics. 2014;15(1):211.

24. Zerbino DR. Using the velvet de novo assembler for short-read sequencing technologies. Curr Protoc Bioinformatics. 2010;11-5.

25. Boetzer M, Pirovano W. Toward almost closed genomes with GapFiller. Genome Biol. 2012;13(6):R56.

26. Kurtz S, Phillippy A, Delcher AL, Smoot M, Shumway M, Antonescu C, Salzberg SL. Versatile and open software for comparing large genomes. Genome Biol. 2004;5(2):R12.

27. Price AL, Jones NC, Pevzner PA. De novo identification of repeat families in large genomes. Bioinformatics. 2005;21:1351-8.

28. Wootton JC, Federhen S. Analysis of compositionally biased regions in sequence databases. Method Enzymol. 1996;266:554-71.

29. Benson G. Tandem repeats finder: a program to analyze DNA sequences. Nucleic Acids Res. 1999;27(2):573-80.

30. Feschotte C, Keswani U, Ranganathan N, Guibotsy ML, Levine D. Exploring repetitive DNA landscapes using REPCLASS, a tool that automates the classification of transposable elements in eukaryotic genomes. Genome Biol Evol. 2009;1:205-20.

31. Ellinghaus $D$, Kurtz S, Willhoeft U. LTRharvest, an efficient and flexible software for de novo detection of LTR retrotransposons. Bmc Bioinformatics. 2008;9:18.

32. Steinbiss $S$, Willhoeft $U$, Gremme G, Kurtz S. Fine-grained annotation and classification of de novo predicted LTR retrotransposons. Nucleic Acids Res. 2009;37(21):7002-13.

33. Kofler R, Schlotterer C, Lelley T. SciRoKo: a new tool for whole genome microsatellite search and investigation. Bioinformatics. 2007;23(13):1683-5.

34. Quinlan AR, Hall IM. BEDTools: a flexible suite of utilities for comparing genomic features. Bioinformatics. 2010;26(6):841-2.

35. Grandaubert J, Bhattacharyya A, Stukenbrock EH. RNA-seq-based gene annotation and comparative genomics of four fungal grass pathogens in the genus Zymoseptoria identify novel orphan genes and species-specific invasions of transposable elements. G3 (Bethesda). 2015;5(7):1323-33.

36. Haas BJ, Zeng Q, Pearson MD, Cuomo CA, Wortman JR. Approaches to fungal genome annotation. Mycology. 2011;2(3):118-41.

37. Haas BJ, Papanicolaou A, Yassour M, Grabherr M, Blood PD, Bowden J, Couger MB, Eccles D, Li B, Lieber M, et al. De novo transcript sequence reconstruction from RNA-seq using the trinity platform for reference generation and analysis. Nat Protoc. 2013;8(8):1494-512.
38. Wu TD, Nacu S. Fast and SNP-tolerant detection of complex variants and splicing in short reads. Bioinformatics. 2010;26(7):873-81.

39. Wu TD, Watanabe CK. GMAP: a genomic mapping and alignment program for mRNA and EST sequences. Bioinformatics. 2005;21(9):1859-75.

40. Haas BJ, Salzberg SL, Zhu W, Pertea M, Allen JE, Orvis J, White O, Buell CR, Wortman JR. Automated eukaryotic gene structure annotation using EVidenceModeler and the program to assemble spliced alignments. Genome Biol. 2008:9(1):R7.

41. Slater GS, Birney E. Automated generation of heuristics for biological sequence comparison. Bmc Bioinformatics. 2005;6:31.

42. Ter-Hovhannisyan V, Lomsadze A, Chernoff YO, Borodovsky M. Gene prediction in novel fungal genomes using an ab initio algorithm with unsupervised training. Genome Res. 2008;18(12):1979-90.

43. Stanke M, Diekhans M, Baertsch R, Haussler D. Using native and syntenically mapped CDNA alignments to improve de novo gene finding. Bioinformatics. 2008;24(5):637-44.

44. Korf I. Gene finding in novel genomes. Bmc Bioinformatics. 2004;5(1):59.

45. Simao FA, Waterhouse RM, loannidis P, Kriventseva EV, Zdobnov EM. BUSCO: assessing genome assembly and annotation completeness with single-copy orthologs. Bioinformatics. 2015;31(19):3210-2.

46. Conesa A, Gotz S, Garcia-Gomez JM, Terol J, Talon M, Robles M. Blast2GO: a universal tool for annotation, visualization and analysis in functional genomics research. Bioinformatics. 2005;21(18):3674-6.

47. Jones P, Binns D, Chang HY, Fraser M, Li WZ, McAnulla C, McWilliam H, Maslen J, Mitchell A, Nuka G, et al. InterProScan 5: genome-scale protein function classification. Bioinformatics. 2014;30(9):1236-40.

48. Yin Y, Mao X, Yang J, Chen X, Mao F, Xu Y. dbCAN: a web resource for automated carbohydrate-active enzyme annotation. Nucleic Acids Res. 2012; 40(Web Server issue):W445-51.

49. Krzywinski M, Schein J, Birol I, Connors J, Gascoyne R, Horsman D, Jones SJ, Marra MA. Circos: an information aesthetic for comparative genomics. Genome Res. 2009;19(9):1639-45.

50. Petersen TN, Brunak S, von Heijne G, Nielsen H. SignalP 4.0: discriminating signal peptides from transmembrane regions. Nat Methods. 2011;8(10):785-6.

51. Emanuelsson O, Brunak S, von Heijne G, Nielsen H. Locating proteins in the cell using TargetP, SignalP and related tools. Nat Protoc. 2007;2(4):953-71.

52. Krogh A, Larsson B, von Heijne G, Sonnhammer ELL. Predicting transmembrane protein topology with a hidden Markov model: application to complete genomes. J Mol Biol. 2001;305(3):567-80.

53. Rawlings ND, Barrett AJ, Finn R. Twenty years of the MEROPS database of proteolytic enzymes, their substrates and inhibitors. Nucleic Acids Res. 2016;44(D1):D343-50.

54. Fawal N, Li Q, Savelli B, Brette M, Passaia G, Fabre M, Mathe C, Dunand C. PeroxiBase: a database for large-scale evolutionary analysis of peroxidases. Nucleic Acids Res. 2013:41(Database issue):D441-4.

55. Urban M, Cuzick A, Rutherford K, Irvine A, Pedro H, Pant R, Sadanadan V, Khamari L, Billal S, Mohanty S, et al. PHI-base: a new interface and further additions for the multi-species pathogen-host interactions database. Nucleic Acids Res. 2017:45(D1):D604-10.

56. Li H: Aligning sequence reads, clone sequences and assembly contigs with BWA-MEM. arXiv:13033997v2[a-bioGN] 2013.

57. Van der Auwera GA, Carneiro MO, Hartl C, Poplin R, Del Angel G, LevyMoonshine A, Jordan T, Shakir K, Roazen D, Thibault J et al: From FastQ data to high confidence variant calls: the genome analysis toolkit best practices pipeline. Curr Protoc Bioinformatics 2013:43:11 10 11-33.

58. Danecek P, Auton A, Abecasis G, Albers CA, Banks E, DePristo MA, Handsaker RE, Lunter G, Marth GT, Sherry ST, et al. The variant call format and VCFtools. Bioinformatics. 2011;27(15):2156-8.

59. Cingolani $P$, Platts $A$, Wang le $L$, Coon M, Nguyen $T$, Wang $L$, Land $S$, Lu X, Ruden DM: A program for annotating and predicting the effects of single nucleotide polymorphisms, SnpEff: SNPs in the genome of Drosophila melanogaster strain w1118; iso-2; iso-3. Fly (Austin) 2012;6(2): 80-92.

60. Li H, Handsaker B, Wysoker A, Fennell T, Ruan J, Homer N, Marth G, Abecasis $\mathrm{G}$, Durbin R, Proc GPD. The sequence alignment/map format and SAMtools. Bioinformatics. 2009;25(16):2078-9.

61. Garrigan D. POPBAM: tools for evolutionary analysis of short read sequence alignments. Evol Bioinforma. 2013;9:343-53.

62. Abyzov A, Urban AE, Snyder M, Gerstein M. CNVnator: an approach to discover, genotype, and characterize typical and atypical CNVs from family and population genome sequencing. Genome Res. 2011;21(6):974-84. 
63. Gruber S, Seidl-Seiboth V. Self versus non-self: fungal cell wall degradation in Trichoderma. Microbiology. 2012;158(Pt 1):26-34.

64. Mittler R, Vanderauwera S, Gollery M, Van Breusegem F. Reactive oxygen gene network of plants. Trends Plant Sci. 2004;9(10):490-8.

65. Tajima F. Statistical-method for testing the neutral mutation hypothesis by DNA polymorphism. Genetics. 1989:123(3):585-95.

66. Rech GE, Sanz-Martin JM, Anisimova M, Sukno SA, Thon MR. Natural selection on coding and noncoding DNA sequences is associated with virulence genes in a plant pathogenic fungus. Genome Biol Evol. 2014:6(9):2368-79.

67. Vitti JJ, Grossman SR, Sabeti PC. Detecting natural selection in genomic data. Annu Rev Genet. 2013;47:97-120.

68. Wicker T, Oberhaensli S, Parlange F, Buchmann JP, Shatalina M, Roffler S, Ben-David R, Dolezel J, Simkova H, Schulze-Lefert P et al: The wheat powdery mildew genome shows the unique evolution of an obligate biotroph. Nat Genet 2013, 45(9):1092-+

69. Shelest E. Transcription factors in fungi. FEMS Microbiol Lett. 2008; 286(2):145-51.

70. Garbelotto MM, Lee HK, Slaughter G, Popenuck T, Cobb FW, Bruns TD. Heterokaryosis is not required for virulence of Heterobasidion annosum. Mycologia. 1997;89(1):92-102.

71. Keriö S, Niemi SM, Haapanen M, Daniel G, Asiegbu FO. Infection of Picea abies clones with a homokaryotic isolate of Heterobasidion parviporum under field conditions. Can J For Res. 2015;45:226-34.

72. Muszewska A, Hoffman-Sommer M, Grynberg M: LTR retrotransposons in Fungi. PLoS One 2011, 6(12).

73. Metzgar D, Bytof J, Wills C. Selection against frameshift mutations limits microsatellite expansion in coding DNA. Genome Res. 2000;10(1):72-80.

74. Gonthier P, Sillo F, Lagostina E, Roccotelli A, Cacciola OS, Stenlid J, Garbelotto M. Selection processes in simple sequence repeats suggest a correlation with their genomic location: insights from a fungal model system. BMC Genomics. 2015;16

75. Karaoglu H, Lee CMY, Meyer W. Survey of simple sequence repeats in completed fungal genomes. Mol Biol Evol. 2005;22(3):639-49.

76. Selker EU. Premeiotic instability of repeated sequences in NeurosporaCrassa. Annu Rev Genet. 1990;24:579-613.

77. Graia F, Lespinet O, Rimbault B, Dequard-Chablat M, Coppin E, Picard M. Genome quality control: RIP (repeat-induced point mutation) comes to Podospora. Mol Microbiol. 2001;40(3):586-95.

78. Idnurm A, Howlett BJ. Analysis of loss of pathogenicity mutants reveals that repeat-induced point mutations can occur in the Dothideomycete Leptosphaeria maculans. Fungal Genet Biol. 2003;39(1):31-7.

79. Rouxel T, Grandaubert J, Hane JK, Hoede C, van de Wouw AP, Couloux A, Dominguez V, Anthouard V, Bally P, Bourras S, et al. Effector diversification within compartments of the Leptosphaeria maculans genome affected by repeat-induced point mutations. Nat Commun. 2011;2:202.

80. Meerupati T, Andersson KM, Friman E, Kumar D, Tunlid A, Ahren D: Genomic mechanisms accounting for the adaptation to parasitism in nematodetrapping Fungi. PLoS Genet 2013, 9(11).

81. Amselem J, Lebrun MH, Quesneville H. Whole genome comparative analysis of transposable elements provides new insight into mechanisms of their inactivation in fungal genomes. BMC Genomics. 2015;16(1):141.

82. Hane JK, Anderson JP, Williams AH, Sperschneider J, Singh KB. Genome sequencing and comparative genomics of the broad host-range pathogen Rhizoctonia solani AG8. PLoS Genet. 2014;10(5):e1004281.

83. Nabel CS, Manning SA, Kohli RM. The curious chemical biology of cytosine: deamination, Methylation,and oxidation as modulators of genomic potential. ACS Chem Biol. 2012;7(1):20-30.

84. Mgbeahuruike AC, Kovalchuk A, Ubhayasekera W, Nelson DR, Yadav JS. CYPome of the conifer pathogen Heterobasidion irregulare: inventory, phylogeny, and transcriptional analysis of the response to biocontrol. Fungal Biol. 2017;121(2):158-71.

85. Chen WP, Lee MK, Jefcoate C, Kim SC, Chen FS, Yu JH. Fungal cytochrome P450 monooxygenases: their distribution, structure, functions, family expansion, and evolutionary origin. Genome Biol Evol. 2014;6(7):1620-34

86. Sezutsu H, Le Goff G, Feyereisen R. Origins of P450 diversity. Philos T R Soc B. 2013;368(1612):20120428.

87. Kubicek CP, Starr TL, Glass NL. Plant Cell Wall-degrading enzymes and their secretion in plant-pathogenic Fungi. Annu Rev Phytopathol. 2014;52:427-51.

88. Seetharam A, Stuart GW. A study on the distribution of 37 well conserved families of $\mathrm{C} 2 \mathrm{H} 2$ zinc finger genes in eukaryotes. BMC Genomics. 2013;14(1):420.
89. Wagner GP, Lynch VJ. The gene regulatory logic of transcription factor evolution. Trends Ecol Evol. 2008;23(7):377-85.

90. Heller J, Tudzynski P. Reactive oxygen species in phytopathogenic fungi: signaling, development, and disease. Annu Rev Phytopathol. 2011:49: 369-90.

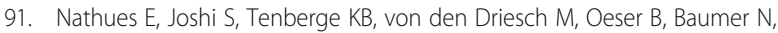
Mihlan M, Tudzynski P. CPTF1, a CREB-like transcription factor, is involved in the oxidative stress response in the phytopathogen Claviceps purpurea and modulates ROS level in its host Secale cereale. Mol Plant Microbe In. 2004;17(4):383-93.

\section{Submit your next manuscript to BioMed Central and we will help you at every step:}

- We accept pre-submission inquiries

- Our selector tool helps you to find the most relevant journal

- We provide round the clock customer support

- Convenient online submission

- Thorough peer review

- Inclusion in PubMed and all major indexing services

- Maximum visibility for your research

Submit your manuscript at www.biomedcentral.com/submit
Biomed Central 